NBER WORKING PAPER SERIES

\title{
MODELING BEHAVIOR DURING A PANDEMIC: USING HIV AS AN HISTORICAL ANALOGY
}

\author{
Nicholas W. Papageorge \\ Working Paper 28898 \\ http://www.nber.org/papers/w28898 \\ NATIONAL BUREAU OF ECONOMIC RESEARCH \\ 1050 Massachusetts Avenue \\ Cambridge, MA 02138 \\ June 2021
}

This manuscript was prepared for the Econometric Society Monograph Series based on a 2020 World Congress talk entitled "Behavior and Policy during a Pandemic: HIV as an Historical Analogy." I am grateful for helpful comments from Jérôme Adda, David Dowdy, Barton Hamilton, Emma Kalish, Eliana La Ferrara, Daniel Polsky and Matthew Zahn along with participants in the 2020 World Congress of the Econometric Society. I also acknowledge excellent research assistance from Emma Kalish and Matthew Zahn. The usual caveats apply. The views expressed herein are those of the author and do not necessarily reflect the views of the National Bureau of Economic Research.

NBER working papers are circulated for discussion and comment purposes. They have not been peer-reviewed or been subject to the review by the NBER Board of Directors that accompanies official NBER publications.

(C) 2021 by Nicholas W. Papageorge. All rights reserved. Short sections of text, not to exceed two paragraphs, may be quoted without explicit permission provided that full credit, including () notice, is given to the source. 
Modeling Behavior during a Pandemic: Using HIV as an Historical Analogy

Nicholas W. Papageorge

NBER Working Paper No. 28898

June 2021

JEL No. I12,I14,I18,I24,J20

\begin{abstract}
$\underline{\text { ABSTRACT }}$
Many models from epidemiology are not designed to capture tradeoffs between health and economic well-being and few are equipped to predict how these tradeoffs interact with individuals' preferences to influence behavior. Policies based on such models may not reflect societal preferences or capture how preferences affect behavior and disease spread. Models with epidemiological processes that do incorporate behavior change or health-wealth tradeoffs (mostly from economics) drastically limit individual-level heterogeneity to remain tractable. This makes it difficult to understand variation in behavioral responses and distributional consequences of the pandemic, which should be central to any evaluation of pandemic-related policy. Without the proper tools, we are unable to fully address even basic policy questions, such as whether the longrun effects of social-distancing justify the costs - and if so, for whom and under what circumstances. After reviewing modeling approaches from epidemiology and economics (among other fields), I propose a framework adapted from earlier work on the HIV/AIDS epidemic. Analysis of individual behavior is based on the idea that health is a form of human capital in which individuals invest through their choices. The model integrates individual level variation that other frameworks omit, but does so at the cost of drastically limiting aggregate economic and epidemiological processes. I conclude that what is needed is a multi-disciplinary effort that helps to bridge gaps across disciplines, which would amount to constructing models that capture key features of different approaches. To be useful in evaluating pandemic-related policy, such a model should have rich heterogeneity and capture how individual choices are linked to the broad contours of disease spread and aggregate economic output. Whether such an effort would be feasible and fruitful remains to be seen.
\end{abstract}

Nicholas W. Papageorge

Department of Economics

Johns Hopkins University

3400 N. Charles Street

Baltimore, MD 21218

and IZA

and also NBER

papageorge@jhu.edu 


\section{Introduction}

The Covid-19 pandemic has affected virtually everyone on the planet, many through illness and death and far more through massive changes in how we live our lives. Many shifts in behavior have stemmed from government policies designed to slow the spread of illness. Others have amounted to individuals' choices to avoid infection. As many countries emerge from the pandemic, the long-run effects of these behavior changes are difficult to predict. We have scarcely begun to fathom the full set of social, cultural and economic consequences of school and business closures, stay-at-home orders, self-quarantine, social distancing and so on. Yet, private efforts to avoid infection and population-wide efforts to slow the pandemic have largely depended on these shifts in behavior. Understanding people's behavior is thus critical for understanding the spread of illness.

Complicating matters, the Covid-19 pandemic, and policies to combat it, have placed uneven burdens on people from different socio-demographic groups.1 This has led to stark variation in behavior, depending on a host of factors that go beyond health risks and government policy to include work arrangements, housing quality and economic resources (Papageorge et al., 2021; Ashraf, 2020; Chiou and Tucker, 2020; Wright et al., 2020). For example, individuals who have been able to comfortably tele-work or who have had access to outside space at home have been more likely to socially distance during the height of the pandemic. Younger and poorer individuals facing lower risk of severe illness, but with little savings, have faced stronger incentives to carry on with their lives, including working in high-risk environments (Gould and Kassa, 2020, Berube and Bateman, 2020). Among other issues, this gives rise to an externality, which absent policy can lead to sub-optimal behavior.

To evaluate potential pandemic-related policies, we need models that can capture variation in individual behavior and its interactions with disease spread and economic well-being. The proximate motivation for developing such tools is the Covid-19 pandemic, but the goal

\footnotetext{
${ }^{1}$ See DeLuca, Papageorge, and Kalish (2020) for early speculation on the uneven burden of the pandemic and measures to control it.
} 
is to consider pandemics more generally. In many countries, widespread vaccinations and declining infection rates have led to the resumption of economic and social activity. However, in many other countries, the pandemic rages on. Moreover, other pandemics may occur, which will be driven by heterogeneous behavioral responses. Designing better policy responses to future pandemics requires the development of better models.

Models from several disciplines capture some critical features of a pandemic, but do so at the cost of omitting others. Many models from epidemiology are not designed to capture tradeoffs between health and economic well-being and few are equipped to predict how these tradeoffs interact with individuals' preferences to affect behavior. Policies based on these models are unlikely to reflect societal preferences or to capture how preferences influence behavior and, thus, disease spread. Models with epidemiological processes that do incorporate behavior change or health-wealth tradeoffs (most from economics) drastically limit individual-level heterogeneity to remain tractable. This makes it difficult to understand variation in behavioral responses and distributional consequences of the pandemic, which should be central to any evaluation of pandemic-related policy.

Given the available set of models, it is difficult to fully address relevant policy questions, such as whether the long-run effects of social-distancing justify the costs - and if so, for whom and under what circumstances. We are in no position to evaluate more complex dynamic tradeoffs, such as whether the short-run benefits of school closures justify the longrun (indeed, inter-generational) costs. Bear in mind that school closures will have outsize effects on the educational trajectories of poor students and, moreover, that low educational attainment is causally linked not only to lower income and employment (Card, 1999), but also to criminal activity (Machin, Marie, and Vujić, 2011), incarceration (Lochner and Moretti, 2004), lack of political engagement (Dee, 2004) and, indeed, poor health and mortality Grossman, 2006). Avoiding the latter two is the goal of closing schools in the first place. This example highlights a general challenge of health policy: while it is true that health undergirds economic well-being, poverty is also deadly. 
This paper explores modeling behavior during a pandemic, relying on two key ideas.

1. Envisioning health as a form of human capital (à la Grossman (1972)) is a useful starting point for understanding what drives variation in individual health decisions. According to the basic framework, individuals invest in their health until the long-run marginal benefits of doing so no longer exceed the upfront costs. Health investments include taking medication, exercising, eating healthy foods or avoiding risky health behaviors, such as drinking excess alcohol or smoking. In the case of a pandemic, avoiding behaviors that raise the probability of infection can be envisioned as a costly investment in health. The framework also links health to employment: health can affect productivity and health investments could interfere with work.

2. While the magnitude of the current crisis seems unprecedented, there are important historical antecedents, most having occurred on a more limited scale, but which nonetheless offer clues concerning behavior change during a pandemic. The HIV/AIDS (Human Immunodeficiency Virus/Acquired Immunodeficiency Syndrome) epidemic in the United States serves as a useful-if imperfect — historical analogy to the current crisis. Lessons learned from this earlier health crisis can increase our understanding of the current one and guide preparations for future pandemics, though with some caveats, which I will discuss below: 2

I begin with a partial review of research on modeling pandemics, focusing on purposes and limitations of different approaches. First, I describe epidemiological models, highlighting how they differ from economic models. While epidemiological models predict disease spread, often taking individual behavior as an input, they are not built to study endogenous behavioral responses to policy or the tradeoffs between public health and the economy. A small subfield of economics known as economic epidemiology attempts to bridge this gap,

\footnotetext{
${ }^{2} \mathrm{I}$ am not the only person to draw a link between these pandemics. If you are reading this, please also read Marcus (2020) and other work by the author. She argues convincingly that shaming people into avoiding risky behavior (both at the height of the AIDS epidemic and during the Covid-19 pandemic) is unlikely to be effective and can backfire since we miss an opportunity to inform people about how to mitigate risk.
} 
though a drawback is that models severely limit individual-level heterogeneity. Second, I discuss research specifically related to Covid-19. The aim is not to cover a massive set of papers from numerous fields, but to how "reduced form" correlational or descriptive empirical studies that evidence rich heterogeneity in behavior are misaligned with models in the economic epidemiological tradition with limited heterogeneity. Third, I detail earlier research estimating structural microeconomic models of individual health decision-making, based on the Grossman framework and applied to an earlier pandemic: HIV/AIDS. These models incorporate many dimensions of heterogeneity that other models miss. However, given that the AIDS epidemic was far less widespread than Covid-19, the models are limited in how they capture aggregate disease spread and do not look at aggregate output at all, suggesting limitations to the HIV analogy.

Next, I introduce a model of behavior during a pandemic adapted from the earlier work on the AIDS epidemic in the United States. The model takes into account limitations to the HIV analogy for the Covid-19 context by specifying aggregate processes for economic output and spread of the virus. The aim is to clarify what a rich microeconomic framework might capture that other models do not. In contrast to epidemiological frameworks, behavior change is endogenous. The Grossman framework is operationalized as a dynamic choice model of risky health behaviors and work choices, which captures a tradeoff between work and health, taking into account that some individuals cannot work while engaging in protective behaviors, such as social distancing. The model also emphasizes rich individual-level heterogeneity (e.g., age, gender, race, socio-economic status, work arrangements and housing quality) in contrast to economic epidemiological models that only allow for some heterogeneity (e.g., young versus old). Finally, in contrast to existing reduced-form work, the model is structural, intended for use in conducting counterfactual policy simulations.

While these features are no doubt desirable, the cost of integrating them is to drastically reduce attention on general equilibrium effects and to exclude many dimensions of heterogeneity used in epidemiological models. Even from a microeconomic perspective, the model 
leaves out many desirable features relevant to policy-making, such as indirect effects of Covid on addiction, mental health and schooling. Moreover, the model would be difficult to estimate given currently available data and thus can help to highlight the kinds of information we ought to collect during future pandemics. In sum, I view the model I present as an initial suggestion of a type of model that has not yet been put forth but should be a part of the discussion on future modeling priorities.

Where does this leave us? We have several classes of models that can be used to develop and evaluate policy related to pandemics. Each captures some features of the pandemic, but omits others. Perhaps that is the best we can do, though it means it is difficult to evaluate the full impacts of pandemic-related policies, such as mask-wearing, restrictions on gatherings and more complex and controversial mandates, such as school closures. To appropriately evaluate resulting tradeoffs, we need better models. A multi-disciplinary effort involving epidemiologists, different flavors of economists and other experts could try to construct a "consensus model," one that incorporates sufficient heterogeneity in individual socio-demographic characteristics and behavior along with the broad contours of the macroeconomy and of disease spread so that policies can be evaluated in light of their short-run and long-run costs and benefits and distributional consequences across socio-demographic groups. $3^{3}$ Should such an effort prove to be futile, a more achievable goal may simply be to write models in each tradition, which means omitting factors deemed important by other fields, but to do so as part of a multi-disciplinary group so that omissions and their consequences for model implications are better understood or more thoughtfully implemented. Any such effort would require serious collaboration, openness to other fields, and an earnest willingness to compromise where possible.

The remainder of this paper is organized as follows. Section 2 reviews literature. Section

\footnotetext{
${ }^{3}$ In this sense, I wholeheartedly agree with suggestions to work together across disciplines, discussed in Murray (2020). Moreover, there are certainly potential cases where policy can improve health and wealth (e.g., loosening mask restrictions as a way to incentivize vaccination). However, given the emphasis I place on recognizing health-wealth tradeoffs, I worry that the author's conclusion, "[W]e need not choose between a healthy public and a healthy economy," downplays the central importance of recognizing such tradeoffs when developing policy.
} 
3 introduces the model. Section 4 concludes.

\section{Literature}

This section provides a broad review of literatures that relate to pandemics. Hopefully, this section makes clear that missing from extant work are models that focus on individual heterogeneity, but that also capture dynamic and aggregate processes of disease spread and economic output, which could be used to study the full impacts of pandemic-related policy.

\subsection{Epidemiology and Economic Epidemiology}

Since the beginning of the Covid-19 pandemic, many researchers from outside epidemiology have become acquainted with the susceptible, infected, and removed (SIR) model. This is a classic framework for modeling infectious diseases first introduced by Kermack and McKendrick (1927). It essentially uses infection rates and the fraction of the population that is susceptible to infection (versus those who are already infected or immune) to predict total infection rates at different points in time. While the model is conceptually straightforward and still underlies more modern methods, it is extremely outdated.

Current models incorporate far more features than the simple SIR model. These include spatial heterogeneity, age-structures and complex networks, among others Martcheva, 2015. Li, Yang, and Martcheva, 2020). These developments reflect increased understanding of how diseases are transmitted and the different ways that susceptible populations increase their risk of infection. As a result of these innovations, models have become increasingly better at predicting disease spread. For example, over the last few years the Centers for Disease Control and Prevention (CDC) have run the "Predict the Influenza Season Challenge" to

foster innovation in modeling and predicting the spread of the flu each year $4^{4}$ One winning team used data assimilation methods commonly employed in numerical weather prediction to

\footnotetext{
${ }^{4}$ Centers for Disease Control and Prevention 2020)
} 
train and optimize a forecasting model for real-time influenza outcomes $5^{5}$ Researchers have produced other forecasting models for diseases such as Ebola, Zika and Covid-19, among many others (Funk et al., 2018, Asher, 2018, Chowell et al., 2016; Roosa et al., 2020).

However, models from epidemiology often share a common assumption. Either explicitly or implicitly, human behavior is seen as fixed, including under counterfactual pandemic circumstances, policies or disease scenarios. In reality, the spread of a disease and policies to combat it affect choices made by individuals and vice versa. Funk et al. (2015) addresses the lack of dynamic behavior in epidemiological models. They identify at least two drawbacks. First, endogenous behavioral change may mean forecasts are unreliable. Second, pandemic mitigation policies often target individual behavior and not the macro-scale dynamics that inform forecasting models. This is a critical disconnect since models are not equipped to evaluate whether individual behavior should be expected to match assumptions about aggregate behavior. Without capturing the endogenous behavioral responses of individuals as the pandemic progresses, epidemiological models omit an important component that ought to inform public health policy. As the authors note, incorporating individual behavior into these epidemiological models is quite challenging and a research frontier that will require cross discipline dialogues. I wholeheartedly agree with their assessment. However, I add that models that omit economic behavior suffer from a additional drawback: they cannot be used to explore tradeoffs between economic and public health consequences of policies to answer questions, such as whether the public health benefits of business closures outweigh the economic costs.

I highlight four sets of papers that incorporate individual behavior into epidemiology. A subfield known as economic epidemiology has made progress in this direction. Gersovitz and Hammer (2004) apply the canonical SIR framework to a representative agent model to examine the externalities of disease prevention and therapy. Fenichel et al. (2011) build a similar framework with agents of varying latent types that make different choices based

\footnotetext{
5 Shaman Group (2014); Centers for Disease Control and Prevention (2014).
} 
on their type. Simulations of their model indicate that shifts in individual behavior have meaningful implications for the course of epidemics. Aadland, Finnoff, and Huang (2013) capture a similar effect in their study of syphilis cycles. The role of group interactions in spreading a disease is studied by Galeotti and Rogers (2013) in the context of strategic immunizations..$^{6}$ Finally, Greenwood et al. (2019) present a model of HIV transmission in Africa to evaluate the effectiveness of public health measures to fight the disease. In contrast to HIV in the United States, HIV in Africa requires more careful incorporation of aggregate effects given that infection rates are much higher. Agents in this model search for different types of sexual relationships, which carry different risks of contracting HIV. Aggregated choices affect how the disease spreads among the population, which in turn affects the expectations of agents when making relationship choices..$^{7}$ In general, the models discussed here facilitate analysis of health-wealth tradeoffs, but the style of model means that heterogeneity remains very limited to maintain tractability. For example, there is often a representative agent, a small number of latent types or 2-3 dimensions of heterogeneity. In contrast, structural micro-economic models, such as those discussed in Section 2.3, allow tens of thousands of ways that individuals can differ.

A second set of papers incorporating behavior into epidemiology appeals to game theoretic concepts $8^{8}$ These models are noteworthy because they allow for policies or changes to the course of the pandemic to affect incentives and thus behavior. Moreover, they allow researchers to formalize and incorporate the idea that individual behavior is not necessarily socially optimal and that society can get stuck in a bad equilibrium given a prisoner's dilemma type of setup (Jentsch, Anand, and Bauch, 2020, McAdams, 2020). These models have been used to study vaccination, in particular the idea that an individual's incentives

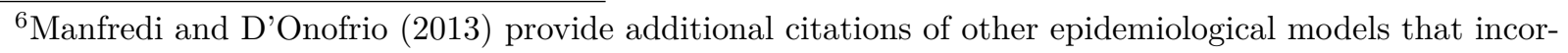
porate some elements of human behavior to model disease spread. Typically, endogenous behavior responses are not directly modeled and heterogeneity is limited.

${ }^{7}$ Similar frameworks are used for Covid-19, which will be discussed in more detail later.

${ }^{8}$ Game theoretic concepts were initially introduced into biological models by Smith and Price $(1973)$ and Taylor and Jonker (1978) as evolutionary stable strategies that described the steady state of a dynamic game.
} 
to become vaccinated decline as vaccination rates rise Bauch, 2005, Yang, Martcheva, and Chen, 2016). While incorporating game theory is clearly a crucial step in the right direction, once again, individual-level heterogeneity remains severely limited in these models and so it is difficult to understand the multiple ways that individuals may react to one another and to policy. Also, to my knowledge, health-wealth tradeoffs remain largely unaddressed in these models.

Third, Adda (2016) builds up a unique epidemiological model of disease transmission that is a function of economic activity. The approach is noteworthy because it incorporates many types of activity (such as school closure and transport networks). The model can be used to relate curtailment of economic activity to disease spread, but also calculates the long-run economic costs. The author finds that reducing interactions effectively controls the spread of a disease but is not cost-effective when looking at the lost economic activity. The framework is applied to influenza, but could be extended to other pandemics and could lead to different policy recommendations. It is possible that a similar model would suggest that policies slowing economic activity are worth the costs in the Covid-19 context. A difference between the framework in Adda (2016) and the framework I present below is that endogenous individual decision-making is not taken into account. Regardless of whether this is a defensible omission in the case of influenza, as earlier literature has shown, it is crucial factor in understanding the spread of Covid-19.

The final set of papers emphasize the importance of heterogeneity as it pertains to health behavior and health policy. Adda (2007) studies how individuals reacted to the "Mad Cow" crisis in the United Kingdom during the late 1990s. Among the questions explored in this paper, the author seeks to understand how new information about susceptibility to this disease affected health-related behaviors and whether there was variation across individuals with differential health risks. The author's analysis finds meaningful heterogeneity in health behavior responses to the risk posed by the Mad Cow crisis. Individuals with low and high susceptibility to the disease did not alter their behaviors. However, those with 
medium susceptibility curb risky behaviors that expose them to the disease but do not completely abandon them. This heterogeneity complicates the government's policy response to addressing health inequalities. Adhvaryu (2014) explores similar challenges in the case of a new Malaria treatment in Tanzania. Misdiagnosis of this disease is common and can introduce short and long run consequences for health and economic well-being. The author documents how Malaria misdiagnoses interact with latent health heterogeneity (i.e., those with Malaria and those without it) leading to slower adoption of new disease treatments that would reap higher returns from adoption. These studies demonstrate the important role of heterogeneity in models of health behavior and policy. Models that omit this feature run the risk of missing important elements driving agent behavior and can imply sub-optimal policy recommendations. Thus, the framework I present below stresses individual heterogeneity.

\subsection{Covid-19 Literature}

Given the rapid proliferation of studies related to Covid-19, it is hard to know where to begin. Many academic communities have turned their attention to the pandemic and it is impossible to do them all justice. First, there is a massive medical, public health and epidemiological literature trying to understand the disease. Medical researchers are focusing on how to treat the disease (Zhu et al., 2020; Beigel et al., 2020; Felsenstein et al., 2020; Wu et al., 2020b); public health scholars are investigating how people are responding to the disease, differences in risk factors, and health disparities brought on by the disease (Bhatraju et al., 2020; Wu et al., 2020a; Lighter et al., 2020; Smith et al., 2020; Rozenfeld et al., 2020, Poulson et al., 2021; Labgold et al., 2021, Ravi, 2020); and epidemiologists are focusing on how the disease will spread (Dowd et al., 2020, Zhang et al., 2020, Ray et al., 2020, Brooks et al., 2020).9 There is also a large literature related to vaccines. Part of this work focuses on how to distribute vaccine resources, which is a question related to medical ethics and

\footnotetext{
${ }^{9}$ Professor Ben Bolker of the Mathematics \& Statistics and Biology Departments at McMaster University has compiled a list of over 30 open source epidemiological models of the Covid-19 pandemic available here.
} 
efficiently combating the disease (Gayle et al., 2020). Others have studied how to optimally develop vaccines for future pandemics. Ahuja et al. (2021) find that if the policy objective is to accelerate vaccine delivery, buyers should directly fund manufacturing capacity and thus shoulder most of the risk of failure, while preserving some direct incentives for speed. Goodkin-Gold et al. (2020) solve for the optimal vaccine subsidy by integrating a vaccine market with rational agents into a standard epidemiological model. They find that the optimal subsidy is non-monotonic in disease infectiousness, peaking for diseases that spread quickly but not so quickly to drive universal vaccination.

Social scientists have also contributed to our understanding of Covid-19. Many have sought to evaluate the effectiveness of measures such as shelter in place orders. Examples include Friedson et al. (2020), Dave et al. (2021), Gupta et al. (2020), Hsiang et al. (2020), Chernozhukov, Kasaha, and Schrimpf (2021), and McLaren and Wang (2020), which use causal inference methods to assess the impact of government interventions, generally showing that a host of measures have been successful at decreasing infections rates and deaths. Others have looked into factors less directly associated with health. Studies such as Barrios and Hochberg (2020), Allcott et al. (2020), and Simonov et al. (2020) have documented a relationship between political affiliations and self-protective behaviors, generally finding conservatives are less likely to adopt these behaviors. While Allcott et al. (2020) incorporates the cost of social distancing into their model, most of these studies do not directly evaluate costs (though they do discuss them when motivating the research).

In general, descriptive and correlational studies of the pandemic have found evidence to suggest there is substantial heterogeneity in behavioral responses across individuals. Papageorge et al. (2021) documents socio-economic behavior gradients for self-protective behaviors such as social distancing. Respondents with higher incomes, flexible work arrangements such as tele-work, and access to open air at home were more likely to adopt these selfprotective behaviors. The authors argue that respondents without these characteristics face a higher cost of adopting these actions, which explains the observed lower levels of take-up. 
Ashraf 2020) explores the relationship between socio-economic factors, government policy and Covid-19 health outcomes using a rich panel data set covering 80 countries. The author finds a strong negative association between Covid-19 cases and socio-economic conditions, which can be alleviated by government policy. Other papers, such as Andersen (2020), Chiou and Tucker (2020), and Wright et al. (2020) rely on cell phone data to track individual behavior during the pandemic. These papers have documented that the adoption of social distancing measures varies across observable characteristics, such as average neighborhood income. Ilin et al. (2020) demonstrate how mobility data can be used to provide real-time feedback to local policymakers on the effectiveness of non-pharmaceutical policy interventions to control the spread of Covid-19 and improve forecast accuracy. The methods proposed by the authors are simple and allow policymakers to recognize heterogeneous responses to policy actions within their communities.

Attempts have also been made by researchers to extend the economic epidemiology models to the Covid-19 pandemic. In addition to describing some of these efforts by other researchers, Atkeson, Kopecky, and Zha (2020, 2021) demonstrate how the SIR framework with endogenous behavior can be used to fit key features of data on Covid-19 deaths in a variety of different geographies. Chang, Martínez, and Velasco (2021) develop a dynamic model of individual location choices in the context of a pandemic by embedding a SIR model of disease spread. In their model a subset of individuals decide how to distribute time in or out of their homes. These choices influence their incomes (taken as exogenous given their choices) and the probability of becoming infected with the disease. The authors calibrate their model and use it to simulate the effects of income transfers (like those from the CARES Act). They find that the cash transfers generate multiple waves of infection as the transfer only temporarily increases the incentive to remain indoors. Farboodi, Jarosch, and Shimer (2020) develop a similar model of dynamic choices with an epidemiological component where forward looking agents choose their level of social interaction. Unlike the models described in the next paragraph, these models include no individual heterogeneity or macroeconomic 
processes.

Other studies add additional economic features to epidemiological models. ${ }^{10}$ For example, Eichenbaum, Rebelo, and Trabandt (2021a), Acemoglu et al. (2020), Brotherhood et al. (2020), and Aguirregabiria et al. (2021) present full-blown macroeconomic models that incorporate epidemiological processes of disease spread.11 Eichenbaum, Rebelo, and Trabandt (2021a) was one of the first models to incorporate the SIR framework into an economic model to study Covid-19. In their model, an economy is populated by identical agents who make choices about how much to consume and work. These choices interact with the SIR features of the model, influencing how the disease spreads and infects others. The model also captures the roles of firms as they produce consumable goods using labor supplied by the agents and the government which can attempt to contain the disease through simple policy measures to limit interactions. Because individuals do not internalize the impact their choices have on the spread of the disease, the competitive equilibrium does not align with the socially optimal outcome 12 The authors simulate the effects of simple policies to contain the spread of the disease, finding that the best containment policy increases the severity of the economic recession but saves a substantial amount of lives ${ }^{13}$ While this model captures many desirable elements (e.g., individual choices, economic production, a host of government policy tools, and disease spread) there is no individual level heterogeneity among the agents.

Determining the optimal pandemic policy is the focus of Acemoglu et al. (2020). The authors extend the classic SIR framework to consider three different age groups of individuals with varying rates of infection, hospitalization, and fatality. In addition, members of each group produce some measure of economic output which is affected by the lockdown policy in

\footnotetext{
${ }^{10}$ For another perspective on the usefulness of these integrated approaches, see Boppart et al. (2020). This paper also includes references to many other economic epidemiological models specifically focused on Covid-19.

${ }^{11}$ Other examples of macro models that incorporate epidemiological processes include Hall, Jones, and Klenow (2020) and Alvarez, Argente, and Lippi (2020).

${ }^{12}$ This type of externality is not unique to Covid-19. Hamilton et al. (2018) find that HIV-infected men who do not use new or experimental medications slow the process of HIV treatment innovation, which negatively affects other patients. As they do not take account of public health impacts of their private choices, there is a negative externality and sub-optimally slow innovation.

13 Eichenbaum, Rebelo, and Trabandt (2021b) incorporates testing into a similar framework.
} 
place. The authors are particularly interested in the policy tradeoff between saving lives and improving economic outcomes. They find that age-targeted polices are welfare enhancing by reducing the amount of deaths and/or economic damage due to the pandemic. Similar to epidemiological models, in the framework, individuals' responses to policies are fixed rather than endogenous. Moreover, heterogeneity is limited to age groups.

Another example is Brotherhood et al. (2020), who present a framework with endogenous behavior choices and disease spread. Their model features old and young agents with varying levels of risk from Covid-19. Agents are able to choose between work and non-work social distancing. These choices influence the probability the agent becomes infected with the disease. Finally, the labor supply decisions and wages of individuals are used to construct a measure of economic output. The authors find that young and old agents make different optimal choices in equilibrium, with older people engaging in substantially more social distancing than the young. Relative to epidemiological models that do not account for endogenous behavior changes, the increase in protective behaviors halves the death rate from the disease. They consider the effects of various policies to fight the pandemic, finding that age specific quarantines and testing schemes may be especially effective at decreasing deaths and increasing individual utility.

Finally, Aguirregabiria et al. (2021) presents a model that captures production and social networks in the context of an infectious disease outbreak. In their framework, individuals choose to work and consume outside or remotely from home. The tradeoff is that work outside is more productive and generates complementarities. On top of these choices, there is a SIR inspired model of disease spread which depends upon (and influences) the network choices of the agents. Work choices are mapped to a production function that not only determines the earnings of individuals, but also the aggregate output of the economy. The authors calibrate this model and perform several policy simulations including ones related to work from home subsidies and testing access. Their analysis finds work from home subsidies are successful in getting individuals to remain at home and control disease spread while 
only modestly increasing the impact of the pandemic recession due to reduced productivity. Increasing testing allows for individuals to confine earlier and exit the in person labor force. These actions simultaneously lower infections and limit the depths of the recession as it is safer for people to work in person at a higher level of productivity.

This summary merely scratches the surface of the depth and breadth of the research into Covid-19. In varying ways, several of the aforementioned papers represent Herculean efforts to merge economics with the epidemiology of Covid-19. Each paper captures economy-wide factors. Yet, each does so at the cost of limiting individual heterogeneity. The first assumes a representative agent, while the second and third allow different age groups. The fourth paper captures individual heterogeneity in terms of their work and consumption networks but not along any socio-demographic dimensions. While age is a crucial distinction in Covid-19, there are many more differences that other literature has shown to be potentially important. Specifically, we are missing a model that captures the tradeoff individuals make between health and economic well-being that also takes into account the well-documented individual heterogeneity in behavioral responses. For example, prolonged policies of social distancing may appear desirable at a high-level but may be incredibly costly for certain groups of people, such as those facing relatively low-risk of serious illness, but who cannot work from home or who live in uncomfortable housing. Policies based on models that assume that levels of compliance do not vary other than by age group are unlikely to be realistic or sustainable. The importance of socio-economic gradients in understanding behavior differences, including responses to pandemic-related policy, is evident from research in the context of HIV/AIDS, which I discuss next.

\subsection{Grossman and Models of the HIV/AIDS Epidemic}

This section focuses on models that apply the Grossman (1972) framework to behavior during the HIV/AIDS epidemic. I present these models in slightly more detail as they serve as a basis for the model of behavior during Covid-19, presented in Section 3. The implicit 
argument is that HIV provides a useful historical analogy for our understanding of Covid-19 and pandemics in general.14

HIV is a virus that reduces the ability of the immune system to fight off routine infections (a condition known as AIDS). It reached epidemic proportions in several countries, including the U.S., starting in 1984. It is transmitted through bodily fluids, most notably through sexual contact. It has disproportionately affected men who have sex with men (MSM) due to relatively high rates of riskier sex behaviors (e.g., multiple partners or not using protection). ${ }^{15}$ Worldwide, it has killed over 32 million people and in the U.S. it has killed more than 700,000.16 Absent treatment, an individual newly infected with HIV lives an average of 11 years and the advent of the AIDS epidemic brought drastic shifts in risky sexual behavior. The early years of the epidemic were quite bleak as treatments were not very effective. A medical breakthrough in 1996 dramatically shifted the prognosis. Known as HAART (highly active anti-retroviral treatment), it reduced mortality rates dramatically. It also made HIV-infected (henceforth $H I V$-positive or $H I V+$ ) people less infectious, which means it also reduced the risk of infection from the perspective of uninfected (henceforth $H I V$-negative or $H I V-)$ people ${ }^{17}$

Applying Grossman (1972) to HIV, behaviors that improve health (or avoidance of risky behavior that diminish health) are viewed as investments. In the models I present, this insight is operationalized as a structural model of dynamic choices that affect people's health, including risky sex behavior and use of medication with side effects. ${ }^{18}$ In specifying these

\footnotetext{
${ }^{14}$ I discuss the limitations of the analogy when motivating the model in Section 3.

${ }^{15}$ Another factor is engagement in anal sex, which has higher rates of infection.

16 Joint United Nations Programme on HIV and AIDS (2020); Kaiser Family Foundation (2019).

17 The next three papers use data from the The Multi-Center AIDS Cohort Study (MACS). MACS is an ongoing longitudinal investigation that has followed a periodically refreshed sample of MSM in four U.S. cities. Descriptive statistics from the data set are in Table 1 for the full sample and then separately for HIV - and HIV + men in both the pre-HAART and the post-HAART eras. Further details on the data set are found in the papers described in this section.

${ }^{18}$ The original framework is theoretical and its insights have often been used in reduced-form analyses in a variety of settings, for example, to examine parental investments in children's health (Currie, 2009). Others have used this insight to study risky health behaviors. Cawley and Ruhm (2011) provide an excellent summary of this literature. Another group of studies, closer to the models presented here, uses the Grossman (1972) framework to build up structural models of health decision-making, which can then be used to evaluate counterfactual policies. This approach has been used to study the timing of medical care decisions, the
} 
models and matching them to data, the goal is to estimate parameters that capture individual preferences governing tradeoffs related to health. Parameters are "structural" in that they are assumed invariant to policy changes, which means estimated models can be used to investigate how behavior would change under counterfactual policy simulations.

\subsubsection{Health, Risky Behavior and the Value of Medical Innovation for Infectious Disease}

In the first paper I discuss applying Grossman (1972) to HIV/AIDS, Chan, Hamilton, and Papageorge (2016), the aim is to examine the value of a medical innovation, HAART, from the perspective of HIV- MSM at risk of becoming infected through their sex decisions. The value of HAART accrues to these individuals in expectation since they only need it if they become positive. Each sexual activity has a corresponding utility and probability of future infection. If infected, agents continue to make decisions based on their own preferences and their health. Medical innovation shifts incentives by lowering mortality and improving health of HIV+ individuals, and also by lowering infection rates, all of which lower the implicit price of risky sex. The model also imposes equilibrium constraints. Infection rates that HIVmen face are in part a function of the optimal behavior of HIV + men. Thus, when they make risky sex decisions, HIV - men must form expectations over infection, which includes the likelihood that their partner is HIV+ given their endogenous sexual behavior. HIVmen also respond to whether or not HIV+ men are using HAART, which lowers infection. Ultimately, the estimated model is used to calculate the lifetime value of HAART and of a counterfactual vaccine. Two key findings emerge. While the value of HAART for HIV - men is far lower than it is for HIV + men, it is substantial (roughly six years of life). Given that the vast majority of MSM are HIV-, roughly half of the total value of HAART accrues, in expectation, to uninfected men who are unlikely to ever need it. Moreover, the model shows

decision to consume alcohol or cigarettes, and use of mental health treatments, among others (Gilleskie 1998, Crawford and Shum, 2005. Chan and Hamilton, 2006. Arcidiacono, Sieg, and Sloan, 2007, Darden 2017; Cronin, Forsstrom, and Papageorge, 2020). 
that the marginal value of a fully functional vaccine is relatively small since HAART already lowered infection rates and lowered the lifetime utility costs of HIV infection.

\subsubsection{How Medical Innovation Creates Value: Health, Human Capital and the Labor Market}

In a second paper, Papageorge (2016), the focus is on another health behavior: use of medication with side effects. The model envisions HIV + individuals as choosing whether or not to work and whether or not to take potentially life saving medications, which may lead to side effects. The model is dynamic because current-period medication choices affect future health and mortality. Moreover, work not only provides income and consumption today, but also leads to higher future earnings through experience. Finally, health and labor interact. Health can affect productivity and thus income, while side effects can influence the disutility of work. The model reveals that side effects play a major role in decision-making because they lower utility directly and make work difficult. Because of how side effects interfere with work, an interesting pattern emerges, which is deemed "optimal treatment cycling." When HIV + men are sick with AIDS, they face a high probability of illness and death, which incentivizes using HAART despite side effects. However, when they recover, the returns to using HAART are lower and so some stop using HAART and return to work. The estimated model is used to examine employment and income assuming HAART was invented as observed and then under a counterfactual innovation: a version of HAART without side effects. A key finding is that lowering side effects not only increases uptake of HAART, but also substantially raises labor supply and income. Broadly, this means that treatment side effects can affect not only health, but also labor market behaviors.

\subsubsection{Innovation and Health Disparities during a Pandemic: The Case of HIV}

In a third paper, Hamilton et al. (2020), the model focuses on behavior differences across socio-demographic groups. As in the earlier paper, HIV + men in the model make medication 
and work decisions while facing side effects, though the focus is on variation in behavior by education group (college or more versus less than college). The estimated model reveals that both education groups take risks by not always using medication with side effects and that both groups respond to incentives, e.g., the uptake of HAART is very high for sick people in both groups. A key difference, however, is that the tradeoff between side effects and health is different across education groups, especially when it comes to work. In particular, individuals in the lower education group are more willing to forgo health to reduce side effects in comparison to their higher-education counterparts. This could be for a number of reasons, including some evidence that the sorts of occupations low education people sort into are less flexible and more physical, which would suggest jobs that are less able to be done with side effects. In other words, people with less education face higher costs of making health investments. A counterfactual non-labor income subsidy decreases employment for all groups. For lower education groups, for whom the health-work tradeoff is starker, it also increases use of HAART and improves health. Broadly, this captures a dynamic that is applicable to Covid-19, a pandemic during which lower-income groups were less likely to engage in self-protecting behaviors in part due to the incompatibility of doing so while remaining employed (Papageorge et al., 2021).

\section{A Grossman Model of Behavior During a Pandemic}

\subsection{Motivation}

In this section, I introduce a discrete choice dynamic model of individual behavior during the Covid-19 pandemic, adapted from the models from the previous section used to study behavior during the AIDS epidemic 19 As with those models, the goal is to identify deep structural parameters governing behavior, in particular, how different individuals face tradeoffs between health and other factors affecting well-being, such as work and income. Armed

\footnotetext{
${ }^{19} \mathrm{~A}$ more elaborate version of the model is found in Hamilton, Papageorge, and Zahn $(2020)$.
} 
with credible estimates, the model could then be used to examine the impact of counterfactual policies on behavior and the spread of illness.

Before introducing the model, I briefly discuss HIV as an analogy for Covid, including limitations. This helps to motivate key model features that make it more applicable to the Covid-19 pandemic, but also to clarify where the model falls short. During both the HIV/AIDS epidemic and the Covid-19 pandemic: (i) we face a widespread, viral pathogen that causes grave illness and death; (ii) costly health investments lower infection risk and also affect how quickly and widely the pathogen spreads; (iii) there is heterogeneity in how people respond to the pandemic and policies to curb it imply different burdens across socio-demographic groups. Thus, as in the HIV/AIDS context, a central feature of a model capturing behavior is a dynamic tradeoff: costly health-related behaviors in the current period can improve future health.

The U.S. AIDS epidemic as an analogy to the current crisis breaks down, however, because HIV affected far fewer individuals and did not cause widespread cessation of economic activity. A model for Covid-19 would need to account for these types of aggregate economic effects. In presenting the framework, I make an initial attempt to incorporate two aggregate processes: disease spread and economic output. These factors are critical components since the aim is to link behavior to the spread of illness and the macroeconomy, where the latter two interact.

It bears mentioning upfront that, while the type of model I present here has several key strengths that should be part of discussions about future modeling priorities, it also sheds light on the work that lies ahead-including data collection-required to build a truly credible and estimable consensus model of a worldwide pandemic. I return to this discussion, which includes specific drawbacks of this model, once I have presented it. 


\subsection{Basic Framework}

\section{Timing}

Prior to period $t$, agent $i$ observes their state variables, which includes the state of the pandemic. The agent forms expectations over the aggregate infection rate and their income shocks. Based on these expectations, the agent makes a joint health and labor supply decision, where the health decision is a costly protective action. 20 The choice is individually optimal given individual $i$ 's beliefs about how these decisions will impact whether they contract the disease (and subsequent consequences). However, aggregate protective actions may affect the course of the pandemic and thus affect other people's utility. Individual $i$ does not take this latter impact into account, which leads to an externality, i.e., choices that are individually optimal may not be socially optimal. After making their choice, the individual realizes their income shocks and consumes. At this point, current-period payoffs accrue. At the end of period $t$, state variables update according to transition processes. By the start of period $t+1$, the agent observes the updated state variables and the process repeats.

\section{Choices}

At time $t$, agent $i$ makes a joint decision regarding protective behaviors and labor supply $d_{i t} \in \mathcal{D} \equiv\{1, \ldots, D\}:$

$$
d_{i t}= \begin{cases}0 & \text { No action taken; Does not work } \\ 1 & \text { No action taken; Works } \\ 2 & \text { Action taken; Does not work } \\ 3 & \text { Action taken; Works }\end{cases}
$$

I assume that there are two different types of agents: low skill and high skill (and these are

\footnotetext{
${ }^{20}$ In the case of HIV, these actions included avoidance of risky sex or use of medications.
} 
assumed fixed over time). Agents with different skills face different choice sets. Namely, low skill workers are not able to choose to take a protective action, such as social distancing, and work. This assumption is intended to reflect the reality that low skill workers may only have access to jobs that are not amenable to social distancing such as retail or gig work. To simplify the model, consumption is treated as an outcome and not a choice, as explained below.

\section{States and Outcomes}

States. The vector of state variables for agent $i$ at time $t$ is denoted as $Z_{i t}$. The set of observed state variables contained in $Z_{i t}$ includes:

$$
\begin{array}{r}
h_{i t} \in\{0,1,2,3,4\}: \text { Health status at } t \\
X_{i t} \in\left\{X^{1}, \ldots, X^{M}\right\}: \text { Non-health state variables at } t \\
\delta_{i t} \in\{0,1\}: \text { Death before } t \\
a_{i t}: \text { Assets at the start of } t \\
\hat{d}_{i t}: \text { History of optimal choices before } t \\
\bar{\lambda}_{t-1}: \text { Aggregate infection rate last period }
\end{array}
$$

During any period $t$, an agent can belong to one of five possible health states:

$$
h_{i t}= \begin{cases}0 & \text { Healthy and not immune } \\ 1 & \text { Infected, asymptomatic, and unaware } \\ 2 & \text { Infected, asymptomatic, and aware } \\ 3 & \text { Infected and symptomatic } \\ 4 & \text { Healthy and immune }\end{cases}
$$


Additional observable state variables are grouped in $X_{i t}$. The $M$ notation reflects the total possible combinations of non-health state variables. Examples of non-health state variables included in $X_{i t}$ are:

1. Agent type $w_{i} \in\{$ low, high $\}$.

2. Age indicator $A_{i t}$, where:

$$
A_{i t}= \begin{cases}0 & \text { if } i \text { is not in an at risk age group at } t \\ 1 & \text { if } i \text { is in an at risk age group at } t\end{cases}
$$

3. Preexisting condition indicator $B_{i t}$, where:

$$
B_{i t}= \begin{cases}0 & \text { if } i \text { does not have a preexisting condition at } t \\ 1 & \text { if } i \text { does have a preexisting condition at } t\end{cases}
$$

4. Government transfer payment $G_{i t}$, where:

$$
G_{i t}= \begin{cases}0 & \text { if } i \text { does not receive a government transfer at } t \\ 1 & \text { if } i \text { receives a government transfer at } t\end{cases}
$$

5. A vector of socio-economic characteristics $S_{i t}$. This object captures individual characteristics such as race, education, home characteristics and living arrangements among others. Reduced form studies of the pandemic such as Papageorge et al. (2021) have documented substantial heterogeneity in behaviors during the pandemic along these characteristics. This framework seeks to capture that same heterogeneity in a structural setting.

The state variable $\delta_{i t}$ measures whether individual $i$ has died from the disease before the beginning of period $t$. The state variable $a_{i t}$ is an indicator for whether individual $i$ has 
three months worth of saved assets or not. The remaining variable in the vector $Z_{i t}$ is $\hat{d}_{t}$, which counts the number of continuous periods in which the agent has adopted protective behaviors up to period $t{ }^{21}$ This is meant to capture either fatigue or growing accustomed to protective actions.

In this model there are aggregate processes that impact an agent's optimal choices. These are the aggregate amount of social distancing across all agents in period $t\left(\bar{d}_{t}\right)$ and the aggregate infection rate in period $t\left(\bar{\lambda}_{t}\right)$. These processes are endogenous to the model and will be discussed in Section 3.4. The agent is able to observe last period's aggregate infection rate $\bar{\lambda}_{t-1}$ as a state variable. Among other factors, the agent uses this information to form expectations over this period's aggregate infection rate when making their optimal choice for period $t$, as will be explained below when we discuss the aggregate processes.

The final component of agent $i$ 's state variables is a vector of choice specific iid utility shocks known to the agent, denoted as $\epsilon_{i t}$. As these are choice specific shocks, the size of this vector is $D$.

Income process ${ }^{22}$ In period $t$ if individual $i$ chooses to work, they earn labor income $y_{i t}$. They also have non-labor income $b_{i t}$, which includes savings and government transfers.

$$
\begin{aligned}
& y_{i t}=f\left(X_{i t}\right)+v_{w t}^{y} \quad v_{w t}^{y} \sim \mathcal{N}\left(0, \sigma_{v^{w}}^{2}\right) \\
& b_{i t}=g\left(X_{i t}\right)+v_{i t}^{b} \quad v_{i t}^{b} \sim \mathcal{N}\left(0, \sigma_{v^{b}}^{2}\right)
\end{aligned}
$$

where $f\left(X_{i t}\right)$ and $g\left(X_{i t}\right)$ are estimated from earnings data. The amount that the agent then consumes $c_{i t}$ is a function of these income sources:

$$
c_{i t}=h\left(y_{i t}, b_{i t}\right)
$$

\footnotetext{
${ }^{21}$ To be clear, if an agent started protective actions at $t=3$ and has continued to do so until $t=5$, then $\hat{d}_{5}=2$. Once an agent stops choosing protective behaviors, this variable will reset to zero until they begin to adopt protective behaviors again.

${ }^{22}$ As mentioned previously, this model is not directly modeling individual consumption and saving decisions. That said, I want to capture elements of these decisions as part of the individual's dynamic problem in this model.
} 
This relationship can also be estimated from data. Within the model the amount an agent consumes depends on their choices which will impact their total income in period $t$. This is a simple way to incorporate consumption as part of the model without imposing the computational burdens of fully modeling consumption and savings in addition to the protective action and labor supply decisions. The limitations brought on by this simplification are perhaps justifiable given that my focus is on the effects that individual protective actions play on aggregate disease spread throughout the population.

\section{Current-Period Payoffs}

Taking measures against an infectious disease requires agents to make sacrifices during the outbreak to prevent getting infected and possible death. The cost of taking these measures will vary by agent based on their observable characteristics and health status. The cost that agent $i$ pays for choice $d=j$ given their health condition $h$ and non-health characteristics $X$ is:

$$
\begin{gathered}
U_{j t}\left(c_{t}, W_{t}, h_{t}, X_{t}, \hat{d}_{t}\right)+\epsilon_{j t}=\log \left(c_{t}\right)+W_{t} \times \theta_{W}+\theta_{h}+A_{t} \times \theta_{A, j}+B_{t} \times \theta_{B, j} \\
+S_{t} \times \theta_{S, j}+\hat{d}_{t} \times \theta_{\hat{d}}+\epsilon_{j t}
\end{gathered}
$$

where the $\log$ operator on $c_{t}$ captures diminishing marginal returns to consumption and $W_{t}$ indicates whether or not the agent chooses to work in period $t$. This setup reflects several points about the costs agents incur during an infectious disease outbreak. First, there is a standalone disutility from supplying labor. Second, their utility parameters are choice specific, which also interact with the agent's observable characteristics. Third, the cost of taking these measures can change if the agent engages in them over multiple periods. 


\subsection{Individual Processes and State-to-State Transitions}

State transitions are especially important in this model. As discussed in Section 3.2, the agent's behaviors are a function of how likely they are to contract the disease according to the prevailing infection rate. These infection rates are also a function of aggregate optimal behavior. I will return to this idea in Section 3.4. In this section, I describe the transition processes for the state variables in the model.

In this model there are two sets of transitions to track.

1. Health status. There are five types of health transitions:

- Healthy to infected: Health state transitions for healthy people are given by $P_{t}\left(h_{t+1}=1 \mid h_{t}=0, X_{t}, d_{t}, \hat{d}_{t}\right)$. This formulation represents this transition probability as a function of the agent's characteristics and their protective action decision. However this does not capture the influence that aggregate social distancing actions $\left(\bar{d}_{t}\right)$ and infection rates $\left(\bar{\lambda}_{t}\right)$ have on the probability that a healthy agent becomes infected. This probability can be reformulated to capture these two effects:

$P_{t}\left(h_{t+1}=1 \mid h_{t}=0, X_{t}, d_{t}, \hat{d}_{t}\right)=\sum_{\bar{h}_{t}=1}^{3} P_{t}\left(h_{t+1}=1 \mid h_{t}=0, X_{t}, d_{t}, \bar{d}_{t}, \bar{\lambda}_{t}\right) \times P_{t}\left(\bar{d}_{t} \mid \bar{h}_{t}\right) P_{t}\left(\bar{\lambda}_{t}\right)$

This specification captures how an individual's health transition depends on their protective action choice, the protective action choice of other people, and the aggregate infection rate. These aggregate processes will be described more in the following section. The first probability on the right-hand side could be taken from data, reduced form estimates or estimated as part of the model. I place an equilibrium constraint on $P_{t}\left(\bar{d}_{t} \mid \bar{h}_{t}\right)$ (i.e., the aggregate choices of other agents in the model) which requires the optimal choices of individuals to be consistent with those predicted by the model. Intuitively, this transition captures the probability 
that a healthy person not taking protective action will interact with a sick person that is also not taking protective action and become infected themselves. Finally, I make the normalization that the probability a healthy agent that engages in social distancing will transition into the unhealthy state is small and denoted as $\omega$.

- Infected, asymptomatic, and unaware to aware: An infected asymptomatic agent becomes aware of their infection status through testing. The probability that an agent receives a test depends on their non-health observable characteristics and is denoted as $\tau\left(X_{i t}\right)$.

○ Infected, asymptomatic to symptomatic: The probability that an infected asymptomatic agent (aware or unaware) becomes symptomatic depends on their nonhealth observable characteristics and is denoted $\zeta\left(X_{i t}\right)$.

- Infected to healthy without immunity: This basic framework does not currently incorporate treatment. Absent this effect, an agent will recover from the disease with some fixed probability. The rate at which an agent recovers from the disease without immunity is denoted as $\psi$. To simplify this transition, $\psi$ will be normalized to 0 if the agent does not choose to take protective actions. This means a sick agent can only recover if they choose to protect themselves.

- Infected to healthy with immunity: The rate at which an agent recovers from the disease with immunity is denoted as $\rho$. For my current purposes, I will assume agents are unable to develop immunity from the disease (i.e., $\rho=0$ ).

2. Death. In this initial model, the probability of death for an infected person is a function of an agent's infection status and non-health characteristics and is denoted as $P\left(\delta_{i t} \mid X_{i t}\right)$. Death is realized at the end of a period. For now, I assume non-infected agents do not die. 


\subsection{Aggregate Processes}

The spread of disease plays important roles in this model. First, individuals respond to disease spread. In the near term, it impacts their current behavior choices. Social distancing may be necessary when the illness is spreading rapidly and the risk of infection is high. As agents look forward, they form expectations for how the disease will continue to spread in the future, which also weighs on their current period behavior choices. Second, individual behavior affects aggregate disease spread. When choosing whether to adopt social distancing, an individual does not internalize the impact of their decision on the overall infection rate, leading to an externality. I have previously addressed the first point. In this section, I discuss the second point about how individual behaviors influence aggregate disease spread.

\section{Aggregate Self-Protective Behaviors}

Aggregate social distancing is summarized by $\bar{d}_{t}$, which is a $5 \times D$ matrix where element $(h, j)$ is the proportion of agents with health status $h$ making choice $j$. This element is denoted $d^{j}\left(h, \bar{\lambda}_{t}, \hat{d}_{t}\right)$ and written as:

$$
d^{j}\left(h, \bar{\lambda}_{t}, \hat{d}_{t}\right)=\sum_{X_{i t}} P_{t}\left(X_{i t} \mid h\right) \times d^{j}\left(X_{i t}, h, \bar{\lambda}_{t}, \hat{d}_{t}\right)
$$

where $P\left(X_{i t} \mid h\right)$ is the heath-state conditional distribution of observed state variables at time

$t$. This expression also depends on the aggregate infection rate $\bar{\lambda}_{t}$, which will be discussed in the next section. The term $d^{j}\left(X_{i t}, h, \bar{\lambda}_{t}, \hat{d}_{t}\right)$ is the probability that an agent will make choice $j$ given the values of the state variables $Z_{i t}$.

\section{Aggregate Infection Rate}

As mentioned previously, the aggregate infection rate in the model is denoted as $\bar{\lambda}_{t}$. To be clear my goal is to build a model of individually optimal behavior that incorporates features of an epidemiological model, not an epidemiological model that forecasts the spread of an 
infectious disease. Thus, our method of modeling the aggregate infection rate will be naive relative to the more sophisticated methods used by epidemiologists. I will start by describing the theory behind the aggregate infection rate, followed by an empirical specification that will be included in the dynamic choice model.

Theory. Consistent with the literature, our model will present a specification for the aggregate infection rate consistent with the classical SIR model. For convenience, I will provide a brief summary of the SIR framework as presented in Gersovitz and Hammer (2004). Start be defining the population within a geographic region $g$ as:

$$
\mathbf{S}+\mathbf{I}+\mathbf{R}=\mathbf{N}
$$

where $\mathbf{S}$ denotes the total number of people in $g$ that are susceptible to the disease, $\mathbf{I}$ is the number of people immune to the disease, and $\mathbf{R}$ is the total number of people that have recovered or died from the disease. These collectively sum to $\mathbf{N}$, the total population. The proportions of these groups in the population are denoted by non-bold versions of the variables: $S, I$, and $R$, where $S+I+R=1$. The continuous time flows for each of these variables is expressed as:

$$
\begin{aligned}
& \dot{\mathbf{N}}=\kappa \mathbf{N}-\delta \mathbf{I} \\
& \dot{\mathbf{S}}=\kappa \mathbf{N}-\alpha \mathbf{S} I+\psi \mathbf{I} \\
& \dot{\mathbf{I}}=\alpha \mathbf{S} I-\mathbf{I}(\psi+\delta+\rho) \\
& \dot{\mathbf{R}}=\rho \mathbf{I}
\end{aligned}
$$

The change in the population rate depends on the birth rate $\kappa$ and the death rate $\delta$ of those infected with the disease. Changes in the susceptible population depend on the inflow of new people, the outflow of susceptible people that have contracted the disease and the inflow of people that have recovered from the disease that are not immune. Here, $\alpha$ captures the rate 
of infection and $\psi$ represents the recovery rate without immunity. Under a random contact assumption, $\mathbf{S} I$ represents the number of susceptible people that were in contact with an infected person. Next, the change in the number of infected people depends on the inflows from the susceptible population, outflows from those that recover but remain susceptible, those that recover from the disease and become immune at a rate $\gamma$, and those that die from the disease. These equations expressed in terms of the population proportions are:

$$
\begin{aligned}
\dot{S} & =(1-S) \kappa+(\delta-\alpha) S I+\psi I \\
\dot{I} & =\alpha S I+\delta I^{2}-I(\kappa+\delta+\psi+\rho) \\
\dot{R} & =\delta I R+\rho I-\kappa R
\end{aligned}
$$

This theoretical framework allows us to specify a process for the aggregate infection rate $\dot{I}$. The key parameter that determines the amount of new cases is $\alpha$. It also directly relates to the choices facing agents in our dynamic discrete choice model. The value of $\alpha$ also likely depends on characteristics of the geographic area such as the population size and density. Similar to Adda (2016) this framework could be extended to decompose $\alpha$ by geography, to capture new cases developed by within geography exposure or cross geography exposure. This will be deferred for now.

Empirical Model. In our empirical model, the aggregate infection rate in region $g$ is denoted as $\bar{\lambda}_{g t}$. The aggregate infection rate represents the number of new cases of the disease, normalized by the size of the population in region $g$. For now, I assume there is only a single geographic region. This simplification means that the growth in infections will only depend on characteristics from within geography $g$. This of course could be expanded to consider multiple geographies and how cross geographic features may influence the spread of the disease. Drawing from the theoretical model and suppressing region notation, we have:

$$
\bar{\lambda}_{t+1}=\alpha S_{t} I_{t}+\delta I_{t}^{2}+\gamma G_{t+1}+\xi_{t+1}
$$


where $S_{t} I_{t}$ is the product of the proportion of the susceptible population and proportion of the infected population in period $t .23$ These proportions are assumed to be analogous to the corresponding elements of $\bar{d}_{t}$, which are denoted as $\tilde{d}\left(h_{t}, j_{t}\right)$. As discussed previously, agents in this model jointly choose protective actions and labor supply. Choices where the agent does not take protective action are denoted as $j_{t}(0)$ and choices where the agent does take protective action are denoted as $j_{t}(1)$. The term $G_{t+1}$ is a vector of geographic characteristics such as population density or geographic fixed effects. Finally, $\xi_{t+1}$ represents the shock, which may be serially correlated during a disease outbreak. In order to address this bias I can introduce an $\mathrm{AR}(1)$ component into the specification. Substituting in for $S_{t}$ and $I_{t}$ along with the $\operatorname{AR}(1)$ we have:

$$
\begin{gathered}
\bar{\lambda}_{t+1}=(1-\phi) \bar{\lambda}_{t}+\alpha_{1} \tilde{d}\left(0, j_{t}(0)\right) \sum_{\bar{h}_{t}=1}^{3} \tilde{d}\left(\bar{h}_{t}, j_{t}(0)\right)+\alpha_{2} \tilde{d}\left(0, j_{t}(1)\right) \sum_{\bar{h}_{t}=1}^{3} \tilde{d}\left(\bar{h}_{t}, j_{t}(0)\right)+ \\
\delta\left(\sum_{\bar{h}_{t}=1}^{3} \tilde{d}\left(\bar{h}_{t}, j_{t}\right)\right)^{2}+\gamma G_{t+1}+\xi_{t+1}
\end{gathered}
$$

There are several features to note about this specification. First, the susceptible population is broken out into two categories. The first is the aggregate proportion of healthy people not taking protective actions $\tilde{d}\left(0, j_{t}(0)\right)$ and the second is the aggregate proportion of healthy people that are taking protective actions $\tilde{d}\left(0, j_{t}(1)\right)$. This formulation allows the infection to spread at different rates across these two susceptible groups (hence the two different $\alpha$ coefficients). The susceptible population can become exposed to the disease by interacting with the infected population who are not taking protective actions, denoted as $\sum_{\bar{h}_{t}=1}^{3} \tilde{d}\left(\bar{h}_{t}, j_{t}(0)\right)$. Second, the parameter $\delta$ in this specification is associated with the death rate from the disease, which impacts anybody infected regardless of their self-protective behavior choice. This value is observable and could be incorporated into the estimation of this process as a constraint. This formulation highlights how the optimal choices of individu-

\footnotetext{
${ }^{23} \mathrm{An}$ implicit assumption in this specification is that the disease incubates for a single period, but this could be altered as needed.
} 
als within the model can impact the aggregate infection rate and subsequent decisions the agents will make. Finally, this specification is flexible enough to allow for the infection rate to vary across the observable characteristics of the agents. This is similar to the approach used by Adda (2016), which estimated different infection rates for various age groups. This procedure would yield infection rates as a function of individual characteristics that could be used in the associated transition probabilities.

As noted above, this specification gets us the spread of the disease as a function of individual behavior through the elements of $\bar{d}_{t}$. By extension this allows us to understand how the spread of the disease depends on the distribution of observable characteristics. In practice, estimates of this process will come from a sample and not the entire population. One can assume as a constraint that I can re-weight as needed so that conditional on the observable characteristics, our sample is random.

\section{Macroeconomic Processes}

The Covid-19 pandemic has highlighted how protective behaviors adopted by individuals and governments to promote public health can have severe effects on the macroeconomy. From our model, it is possible to construct measures of employment and output. For example, following the approach used in Brotherhood et al. (2020), GDP in period $t$ could be calculated by summing the wage rate multiplied by the amount of time the individual spent working:

$$
Y_{t}=\sum_{w} \sum_{i} W_{i t} y_{i t}^{w}
$$

A similar approach could be used to calculate the unemployment rate of individuals in the model. These methods are straightforward but other approaches could be adopted. A further extension would be to allow these macroeconomic processes to influence other elements of the model. For instance, if the economy enters a recession, the amount of jobs or wages available to the individuals could be impacted. These effects would influence the optimal work and 
consumption choices agents make within the dynamic model but substantially increase the complexity of the model. Eichenbaum, Rebelo, and Trabandt (2021a) is an example of a model that endogenizes wages. In the model wages are not only impacted by the choice of firms but also the spread of the disease. However, as discussed previously, this model includes no individual level heterogeneity apart from the agent's infection status.

\subsection{The Value Function and Choice Probabilities}

For an agent that has not died before period $t$, their value function for choice $j \in \mathcal{D}$ is:

$$
\begin{aligned}
& V_{j t}\left(Z_{t}\right)=U_{j t}\left(c_{t}, W_{t}, h_{t}, X_{t}, \hat{d}_{t}\right)+\epsilon_{j t}+ \\
& \quad \sum_{Z_{t+1}} f_{j t}\left(Z_{t+1} \mid Z_{t}, \bar{d}_{t}, \bar{\lambda}_{t}\right) \times \beta\left[E_{\epsilon}\left(\max _{k}\left\{V_{k t+1}\left(Z_{t+1}\right)+\epsilon_{k t+1}\right\}\right) \times\left(1-\delta_{t+1}\right)+v \times \delta_{t+1}\right]
\end{aligned}
$$

Where $U_{j t}\left(c_{t}, W_{t}, h_{t}, X_{t}, \hat{d}_{t}\right)+\epsilon_{j t}$ represents the agent's current period payoffs, $f_{j t}\left(Z_{t+1} \mid Z_{t}, \bar{d}_{t}, \bar{\lambda}_{t}\right)$ represents the probability distribution over future values of the state variables, and $\beta$ is the discount factor over future choices. The term $v$ represents a "scrap value" of life parameter that could be estimated or normalized to zero ${ }^{24}$ The objects of the model are summarized in Table 2 ,

\section{Choice Probabilities in Equilibrium}

Given the normalizations and assumptions of the model, the net-of-errors value function for choice $j$ for agent $i$ is:

$$
\begin{aligned}
\bar{V}_{j t}\left(Z_{t}\right)=U_{j t}\left(c_{t}, W_{t}, h_{t}, X_{t}, \hat{d}_{t}\right) & +\sum_{Z_{t+1}} f_{j}\left(Z_{t+1} \mid Z_{t}, \bar{d}_{t}, \bar{\lambda}_{t}\right) \\
& \times \beta\left[\left(\varsigma+\log \left[\sum_{k}^{D} \exp \left(\bar{V}_{k t+1}\left(Z_{t+1}\right)\right)\right]\right) \times\left(1-\delta_{t+1}\right)\right]
\end{aligned}
$$

Euler's constant is denoted by $\varsigma$. Given the error term assumptions, the conditional choice

\footnotetext{
${ }^{24}$ For the remainder of this discussion, I will proceed with latter approach.
} 
probability for choice $j$ is:

$$
\phi_{j t}\left(Z_{t}\right)=\frac{\exp \left(\bar{V}_{j t}\left(Z_{t}\right)\right)}{\sum_{k}^{D} \exp \left(\bar{V}_{k t}\left(Z_{t}\right)\right)}
$$

Similar to Chan, Hamilton, and Papageorge (2016) there will be an equilibrium constraint incorporated into the health transition from healthy to infected. This transition probability can be expressed as:

$$
P_{t}\left(h_{t+1}=1 \mid h_{t}=0, X_{t}, d_{t}, \hat{d}_{t}\right)=P_{t}\left(h_{t+1}=1 \mid h_{t}=0, X_{t}, d_{t}, \bar{d}_{t}, \bar{\lambda}_{t}\right) \times d^{j}\left(h_{t}, \bar{\lambda}_{t}, \hat{d}_{t}\right) \bar{\lambda}_{t}
$$

where $P_{t}\left(\bar{d}_{t} \mid \bar{h}_{t}\right)=d^{j}\left(h_{t}, \bar{\lambda}_{t}, \hat{d}_{t}\right)$ and $P_{t}\left(\bar{\lambda}_{t}\right)=\bar{\lambda}_{t}$. When defining this expression, I have assumed that the probability of choosing $d$ conditional on health status $h$ comes from the $\bar{d}_{t}$ matrix, whose $(h, j)$ element is expressed as:

$$
d^{j}\left(h, \bar{\lambda}_{t}, \hat{d}_{t}\right)=\sum_{X_{i t}} P_{t}\left(X_{i t} \mid h\right) \times d^{j}\left(X_{i t}, h, \bar{\lambda}_{t}, \hat{d}_{t}\right)
$$

The equilibrium constraint is that the individual choice probabilities in this expression are equal to the optimal choice probabilities implied by the model:

$$
d^{j}\left(X_{i t}, h, \bar{\lambda}_{t}, \hat{d}_{t}\right)=\phi_{j t}\left(Z_{t}\right)
$$

Defining the vector of structural parameters in the model as $\Xi$, the optimal choice probabilities when the market is at equilibrium are:

$$
\phi_{j t}\left(Z_{t} ; \Xi\right)=\frac{\exp \left(\bar{V}_{j t}\left(Z_{t}, \phi_{j t}\left(Z_{t} ; \Xi\right)\right)\right)}{\sum_{k}^{D} \exp \left(\bar{V}_{k t}\left(Z_{t}, \phi_{k t}\left(Z_{t} ; \Xi\right)\right)\right)}
$$

As a result of this equilibrium constraint, the structural parameters will not only influence an agent's optimal choice, but also how they respond to the optimal equilibrium behavior of 
other agents. It is important to note that there may be multiple equilibria in these choice probabilities. For instance, consider the interpretation of protective actions as wearing a mask. If mask wearing throughout the population increases, any given individual is safer from the disease and may find it optimal for them to not engage in this behavior. Alternatively, if mask wearing has become commonplace a social norm may have developed around this behavior. As such, it may be costlier for an individual to not engage in this action and they continue to wear masks despite facing less risk from the disease. Finally, Appendix 6 addresses the estimation procedure and data requirements for the model.

\subsection{Discussion of the Model}

As specified, the model I present is problematic. It is not clear whether it is tractable enough to solve and to estimate. Among other things, there may be multiple equilibria, which makes it difficult to credibly conduct counterfactual policy simulations. Nor is it clear if aggregate processes are sufficiently captured to make this model useful for policy analysis in the context of a worldwide pandemic. Moreover, data limitations could thwart efforts to identify key parameters. Even now, over a year into the Covid-19 pandemic, we lack basic figures, such as the number of people who have been infected (versus the number who have merely tested positive). Finally, while the model provides richer individual-level heterogeneity compared to existing models in the economic epidemiological tradition, it contains far fewer variables related to disease spread compared to standard models from epidemiology. Even by the standards of structural microeconomic models, this model omits many crucial factors that limit its usefulness and could also lead to biased estimates of key parameters capturing tradeoffs. For example, the model is silent on addiction or mental health issues, both of which could influence behavior during the pandemic and would likely be subsumed in the various "cost" parameters. Among other issues, this means such parameters may not be fixed under counterfactual policies. Thus, while this model offers some nice features that other models lack, it falls short on many dimensions. It is best viewed as an initial suggestion 
of some of the tradeoffs we should be considering more carefully in any discussion of future modeling priorities and attempts to develop more helpful tools.

\section{Concluding Remarks: What Lies Ahead?}

To date, we lack models of behavior during a pandemic that incorporate rich heterogeneity in how people approach health-wealth tradeoffs, and which credibly capture aggregate processes such as economic growth and disease spread. It is therefore difficult to answer many relevant policy questions. Consider the following: What is the optimal proportion of the population that should avoid contact with anyone outside their household given a local rate of infection? Messaging and politics aside, the number is very unlikely to be 0 or 100 . Even if society decided that public health is our only concern, the optimal number is still not 100 because economic downturns have all sorts of negative consequences that affect health directly (e.g., addiction and abuse) and indirectly (e.g., job losses and lower educational attainment). Even if economic well-being were the only concern, the number would not be 0 because health affects the economy in many complex ways.

Whether a consensus model or a set of consensus models can be developed that is reasonably well-equipped to answer such policy questions is not clear. The likelihood may not be terribly high, though if no attempt is made to reach across disciplines to discuss modeling priorities, the likelihood is zero. Thus, my first suggestion is deliberate dialogue across disciplines. Even if that does not lead to an agreed-upon consensus model or set of models, it might clarify what each set of models ought and ought not be used to do. It may also highlight what sorts of data ought to be collected during a pandemic (e.g., from randomized testing so we have some idea of how many infections have gone undetected) and which modeling features are deemed most or least crucial by different disciplines. That is a place to start.

Absent such an attempt, my fear is that we will continue to develop policy without the 
right modeling tools, essentially asking models from one field or another to provide guidance in ways they are not designed to, which leads to non-credible policy. Alternatively, we make policy without science, which opens the door for politics, wishful thinking, morality or conspiracies to drive policy-making. Depressingly, the HIV/AIDS context once again provides an historical analogy. Unrealistic "abstinence only" messages ignored basic human real, daily and pressing tradeoffs that people faced. Such messaging also limited opportunities to provide information to mitigate risk (Marcus, 2020). Similarly "all-or-nothing" approaches have abounded during Covid-19. Recent reporting has indicated that allowing 20\% capacity at bars and restaurants could maintain over half of visits and decrease infections by nearly 90\%. Sadly, it took until December of 2020 for this point to be made in a newspaper 25 Appropriate scientific modeling of behavior and the virus could have clarified these tradeoffs much earlier 26

Covid-19 vaccinations in the U.S. are well-underway so there is some light at the end of this particular tunnel (Neergaard, 2020). However, there are reasons to continue the project of building appropriate models for this pandemic. They can help us to understand which policies during Covid-19 have been most helpful and which have been unduly costly and harmful. They can also shed light on distributional consequences, the repercussions of which will be with us for years to come. In the case of Covid-19, this work has only just begun. Scholars continue to provide evidence of the economic, social and cultural shifts from the height of the U.S. AIDS epidemic (White, 1991; Godlonton, Munthali, and Thornton, 2016 Fernández, Parsa, and Viarengo, 2019; Papageorge et al. 2019). Moreover, It is only a matter of time before the next pandemic arrives. When it does, we will want to have achieved a better understanding of human behavior during a pandemic, including socio-demographic differences in behavior and the role of health-wealth tradeoffs along with the distributional

\footnotetext{
${ }^{25}$ Similarly, the likelihood of HIV transmission varies substantially across sexual behaviors (Varghese et al. 2002). Providing this type of information early on and clearly might have allowed people to make more informed choices, lowering the spread of infection while recognizing that intimacy is a fundamental part of people's lives (Godlonton, Munthali, and Thornton, 2016)

${ }^{26}$ See Serkez $(2020)$.
} 
consequences of the pandemic and of policies to curb it. Tools developed to analyze the current pandemic could then be tailored to evaluate policies related to the next crisis.

\section{References}

Aadland, David, David C. Finnoff, and Kevin X.D. Huang. 2013. "Syphilis Cycles." The B.E. Journal of Economic Analysis and Policy 14 (1):297-348.

Acemoglu, Daron, Victor Chernozhukov, Iván Werning, and Michael D. Whinston. 2020. "A Multi-Rosk SIR Model with Optimally Targeted Lockdown." Working Paper 27102, National Bureau of Economic Research.

Adda, Jérôme. 2007. "Behavior Towards Health Risks: An Empirical Study Using the "Mad Cow" Crisis as an Experiment." Journal of Risk and Uncertainty 35 (3):285-305.

. 2016. "Economic Activity and the Spread of Viral Diseases: Evidence from High Frequency Data." The Quarterly Journal of Economics 131 (2):891-941.

Adhvaryu, Achyuta. 2014. "Learning, Misallocation, and Technology Adoption: Evidence From New Malaria Therapy in Tanzania." The Review of Economic Studies 81 (4):13311365.

Aguirregabiria, Victor, Jiaying Gu, Yao Luo, and Pedro Mira. 2021. "Diffusion of Covid-19 in Social and Production Networks: Simulation Evidence from a Dynamic Model." Annals of Economics and Statistics Forthcoming.

Ahuja, Amrita, Susan Athey, Arthur Baker, Eric Budish, Juan Camilo Castillo, Rachel Glennerster, Scott Duke Kominers, Michael Kremer, Jean Lee, Canice Prendergast, Christopher M. Snyder, Alex Tabarrok, Brandon Joel Tan, and Witold Więcek. 2021. "Preparing for a Pandemic: Accelerating Vaccine Availability." Working Paper 28492, National Bureau of Economic Research.

Allcott, Hunt, Levi Boxell, Jacob Conway, Matthew Gentzkow, Michael Thaler, and David Y. Yang. 2020. "Polarization and Public Health: Partisan Differences in Social Distancing During the Coronavirus Pandemic." Journal of Public Economics 191:104254.

Alvarez, Fernando E., David Argente, and Francesco Lippi. 2020. "A Simple Planning Problem for COVID-19 Lockdown." Working Paper 26981, National Bureau of Economic Research.

Andersen, Martin. 2020. "Early Evidence on Social Distancing in Response to COVID-19 in the United States." Working Paper 3569368, SSRN.

Arcidiacono, Peter, Holger Sieg, and Frank Sloan. 2007. "Living Rationally Under the Volcano? An Empirical Analysis of Heavy Drinking and Smoking." International Economic Review 48 (1):37-65. 
Asher, Jason. 2018. "Forecasting Ebola with a Regression Transmission Model." Epidemics $22: 50-55$.

Ashraf, Badar Nadeem. 2020. "Socioeconomic Conditions, Government Interventions and Health Outcomes During COVID-19." Covid Economics 37:141-162.

Atkeson, Andrew G., Karen Kopecky, and Tao Zha. 2020. "Behavior and the Transmission of Covid-19." Mimeo, University of California Los Angeles.

. 2021. "Behavior and the Transmission of Covid-19." AEA Papers and Proceedings 111:356-60.

Barrios, John M. and Yael Hochberg. 2020. "Risk Perception Through the Lens of Politics in the Time of the COVID-19 Pandemic." Working Paper 27008, National Bureau of Economic Research.

Bauch, Chris T. 2005. "Imitation Dynamics Predict Vaccinating Behaviour." Proceedings of the Royal Society B: Biological Sciences 272 (1573):1669-1675.

Beigel, John H., Kay M. Tomashek, Lori E. Dodd, Aneesh K. Mehta, Barry S. Zingman, Andre C. Kalil, Elizabeth Hohmann, Helen Y. Chu, Annie Luetkemeyer, Susan Kline, Diego Lopez de Castilla, Robert W. Finberg, Kerry Dierberg, Victor Tapson, Lanny Hsieh, Thomas F. Patterson, Roger Paredes, Daniel A. Sweeney, William R. Short, Giota Touloumi, David Chien Lye, Norio Ohmagari, Myoung-don Oh, Guillermo M. RuizPalacios, Thomas Benfield, Gerd Fätkenheuer, Mark G. Kortepeter, Robert L. Atmar, C. Buddy Creech, Jens Lundgren, Abdel G. Babiker, Sarah Pett, James D. Neaton, Timothy H. Burgess, Tyler Bonnett, Michelle Green, Mat Makowski, Anu Osinusi, Seema Nayak, and H. Clifford Lane. 2020. "Remdesivir for the Treatment of Covid-19 — Final Report." New England Journal of Medicine 383 (19):1813-1826.

Berube, Alan and Nicole Bateman. 2020. "Who Are the Workers Already Impacted by the COVID-19 Recession?" Metropolitan Policy Program Covid-19 Analysis, Brookings Institute.

Bhatraju, Pavan K., Bijan J. Ghassemieh, Michelle Nichols, Richard Kim, Keith R. Jerome, Arun K. Nalla, Alexander L. Greninger, Sudhakar Pipavath, Mark M. Wurfel, Laura Evans, Patricia A. Kritek, T. Eoin West, Andrew Luks, Anthony Gerbino, Chris R. Dale, Jason D. Goldman, Shane O'Mahony, and Carmen Mikacenic. 2020. "Covid-19 in Critically Ill Patients in the Seattle Region - Case Series." New England Journal of Medicine 382 (21):2012-2022.

Boppart, Timo, Karl Harmenberg, John Hassler, Per Krusell, and Jonna Olsson. 2020. Confronting Epidemics: The Need for Epi-econ IAMs. Sweden: Konjunkturinstitutet.

Brooks, Logan C., Evan L. Ray, Jacob Bien, Johannes Bracher, Aaron Rumack, Ryan J. Tibshirani, and Nicholas G. Reich. 2020. "Comparing Ensemble Approaches for ShortTerm Probabilistic COVID-19 Forecasts in the U.S." Forecasting News, International Institute of Forecasters. 
Brotherhood, Luiz, Philipp Kircher, Cezar Santos, and Michèle Tertilt. 2020. "An Economic Model of the Covid-19 Pandemic with Young and Old Agents: Behavior, Testing and Policies." Discussion Paper 175, Collaborative Research Center Transregio 224.

Card, David. 1999. "The Causal Effect of Education on Earnings." In Handbook of Labor Economics, vol. 3. Elsevier, 1801-1863.

Cawley, John and Christopher J Ruhm. 2011. "The Economics of Risky Health Behaviors." In Handbook of Health Economics, vol. 2. Elsevier, 95-199.

Centers for Disease Control and Prevention. 2014. "CDC Announces Winner of the "Predict the Influenza Season Challenge." Available at https://www.cdc.gov/flu/news/ predict-flu-challenge-winner.htm.

. 2020. "FluSight: Flu Forecasting." Available at https://www.cdc.gov/flu/ weekly/flusight/index.html.

Chan, Tat Y. and Barton H. Hamilton. 2006. "Learning, Private Information, and the Economic Evaluation of Randomized Experiments." Journal of Political Economy 114 (6):9971040.

Chan, Tat Y., Barton H. Hamilton, and Nicholas W. Papageorge. 2016. "Health, Risky Behaviour and the Value of Medical Innovation for Infectious Disease." Review of Economic Studies 83 (4):1465-1510.

Chang, Roberto, Humberto Martínez, and Andrés Velasco. 2021. "Pandemics, Incentives, and Economic Policy: A Dynamic Model." Working Paper 28636, National Bureau of Economic Research.

Chernozhukov, Victor, Hiroyuki Kasaha, and Paul Schrimpf. 2021. "Causal Impact of Masks, Policies, Behavior on Early COVID-19 Pandemic in the U.S." Journal of Econometrics 220 (1):23-62.

Chiou, Lesley and Catherine Tucker. 2020. "Social Distancing, Internet Access and Inequality." Working Paper 26982, National Bureau of Economic Research.

Chowell, Gerardo, Doracelly Hincapie-Palacio, Juan Ospina, Bruce Pell, Amna Tariq, Sushma Dahal, Seyed Moghadas, Alexandra Smirnova, Lone Simonsen, and Cécile Viboud. 2016. "Using Phenomenological Models to Characterize Transmissibility and Forecast Patterns and Final Burden of Zika Epidemics." Public Library of Science Currents 8.

Crawford, Gregory S. and Matthew Shum. 2005. "Uncertainty and Learning in Pharmaceutical Demand." Econometrica 73 (4):1137-1173.

Cronin, Christopher J., Matthew P. Forsstrom, and Nicholas W. Papageorge. 2020. "What Good Are Treatment Effects Without Treatment? Mental Health and the Reluctance to Use Talk Therapy." Working Paper 27711, National Bureau of Economic Research. 
Currie, Janet. 2009. "Healthy, Wealthy, and Wise: Socioeconomic Status, Poor Health in Childhood, and Human Capital Development." Journal of Economic Literature 47 (1):87122.

Darden, Michael. 2017. "Smoking, Expectations, and Health: A Dynamic Stochastic Model of Lifetime Smoking Behavior." Journal of Political Economy 125 (5):1465-1522.

Dave, Dhaval, Andrew I. Friedson, Kyutaro Matsuzawa, and Joseph J. Sabia. 2021. "When Do Shelter-in-Place Orders Fight Covid-19 Best? Policy Heterogeneity Across States and Adoption Time." Economic Inquiry 59 (1):29-52.

Dee, Thomas S. 2004. "Are There Civic Returns to Education?" Journal of Public Economics 88 (9):1697-1720.

DeLuca, Stephanie, Nicholas W. Papageorge, and Emma Kalish. 2020. "The Unequal Cost of Social Distancing." Johns Hopkins University Coronavirus Resource Center. Available at https://coronavirus.jhu.edu/from-our-experts/ the-unequal-cost-of-social-distancing.

Dowd, Jennifer Beam, Liliana Andriano, David M. Brazel, Valentina Rotondi, Per Block, Xuejie Ding, Yan Liu, and Melinda C. Mills. 2020. "Demographic Science Aids in Understanding the Spread and Fatality Rates of COVID-19." Proceedings of the National Academy of Sciences 117 (18):9696-9698.

Eichenbaum, Martin S., Sergio Rebelo, and Mathias Trabandt. 2021a. "The Macroeconomics of Epidemics." Working Paper 26882, National Bureau of Economic Research.

. 2021b. "The Macroeconomics of Testing and Quarantining." Working Paper 27104, National Bureau of Economic Research.

Farboodi, Maryam, Gregor Jarosch, and Robert Shimer. 2020. "Internal and External Effects of Social Distancing in a Pandemic." Working Paper 27059, National Bureau of Economic Research.

Felsenstein, Susanna, Jenny A. Herbert, Paul S. McNamara, and Christian M. Hedrich. 2020. "COVID-19: Immunology and Treatment Options." Clinical Immunology 215:108448.

Fenichel, Eli P., Carlos Castillo-Chavez, M. G. Ceddia, Gerardo Chowell, Paula A. Gonzalez Parra, Graham J. Hickling, Garth Holloway, Richard Horan, Benjamin Morin, Charles Perrings, Michael Springborn, Leticia Velazquez, and Cristina Villalobos. 2011. "Adaptive Human Behavior in Epidemiological Models." Proceedings of the National Academy of Sciences 108 (15):6306-6311.

Fernández, Raquel, Sahar Parsa, and Martina Viarengo. 2019. "Coming out in America: AIDS, Politics, and Cultural Change." Working Paper 25697, National Bureau of Economic Research. 
Friedson, Andrew I, Drew McNichols, Joseph J Sabia, and Dhaval Dave. 2020. "Did California's Shelter-in-Place Order Work? Early Coronavirus-Related Public Health Effects." Working Paper 26992, National Bureau of Economic Research.

Funk, Sebastian, Shweta Bansal, Chris T. Bauch, Ken T.D. Eames, W. John Edmunds, Alison P. Galvani, and Petra Klepac. 2015. "Nine Challenges in Incorporating the Dynamics of Behaviour in Infectious Diseases Models." Epidemics 10:21-25.

Funk, Sebastian, Anton Camacho, Adam J Kucharski, Rosalind M Eggo, and W John Edmunds. 2018. "Real-Time Forecasting of Infectious Disease Dynamics with a Stochastic Semi-Mechanistic Model." Epidemics 22:56-61.

Galeotti, Andrea and Brian W. Rogers. 2013. "Strategic Immunization and Group Structure." American Economic Journal: Microeconomics 5 (2):1-32.

Gayle, Helene, William Foege, Lisa Brown, and Benjamin Kahn, editors. 2020. Framework for Equitable Allocation of COVID-19 Vaccine. Washington, DC: The National Academies Press.

Gersovitz, Mark and Jeffrey S. Hammer. 2004. "The Economical Control of Infectious Diseases." The Economic Journal 114 (492):1-27.

Gilleskie, Donna B. 1998. "A Dynamic Stochastic Model of Medical Care Use and Work Absence." Econometrica 66 (1):1-45.

Godlonton, Susan, Alister Munthali, and Rebecca Thornton. 2016. "Responding to Risk: Circumcision, Information, and HIV Prevention." Review of Economics and Statistics 98 (2):333-349.

Goodkin-Gold, Matthew, Michael Kremer, Christopher M. Snyder, and Heidi L. Williams. 2020. "Optimal Vaccine Subsidies for Endemic and Epidemic Diseases." Working Paper 28085, National Bureau of Economic Research.

Gould, Elise and Melat Kassa. 2020. "Young Workers Hit Hard by the COVID-19 Economy." White Paper, Economic Policy Institute.

Greenwood, Jeremy, Philipp Kircher, Cezar Santos, and Michele Tertilt. 2019. "An Equilibrium Model of the African HIV/AIDS Epidemic." Econometrica 87 (4):1081-1113.

Grossman, Michael. 1972. "On the Concept of Health Capital and the Demand for Health." Journal of Political Economy 80 (2):223-255.

- 2006. "Education and Nonmarket Outcomes." In Handbook of the Economics of Education, Handbooks in Economics, vol. 1, edited by Eric A. Hanushek and Finis Welch. Elsevier, 577-633.

Gupta, Sumedha, Thuy D Nguyen, Felipe Lozano Rojas, Shyam Raman, Byungkyu Lee, Ana Bento, Kosali I Simon, and Coady Wing. 2020. "Tracking Public and Private Response to the Covid-19 Epidemic: Evidence from State and Local Government Actions." Working Paper 27027, National Bureau of Economic Research. 
Hall, Robert E., Charles I. Jones, and Peter J. Klenow. 2020. "Trading Off Consumption and COVID-19 Deaths." Working Paper 27340, National Bureau of Economic Research.

Hamilton, Barton H., Andres Hincapie, Emma C. Kalish, and Nicholas W. Papageorge. 2020. "Innovation and Health Disparities during a Pandemic: The Case of HIV." Mimeo, Johns Hopkins University.

Hamilton, Barton H., Andrés Hincapié, Robert A. Miller, and Nicholas W. Papageorge. 2018. "Innovation and Diffusion of Medical Treatment." Working Paper 24577, National Bureau of Economic Research.

Hamilton, Barton H., Nicholas W. Papageorge, and Matthew V. Zahn. 2020. "Modeling an Infectious Disease from an Individual Dynamic Discrete Choice Perspective." Mimeo, Johns Hopkins University.

Hsiang, Solomon, Daniel Allen, Sébastien Annan-Phan, Kendon Bell, Ian Bolliger, Trinetta Chong, Hannah Druckenmiller, Luna Yue Huang, Andrew Hultgren, Emma Krasovich et al. 2020. "The Effect of Large-Scale Anti-Contagion Policies on the COVID-19 Pandemic." Nature 584 (7820):262-267.

Ilin, Cornelia, Sébastien E. Annan-Phan, Xiao Hui Tai, Shikhar Mehra, Solomon M. Hsiang, and Joshua E. Blumenstock. 2020. "Public Mobility Data Enables COVID-19 Forecasting and Management at Local and Global Scales." Working Paper 28120, National Bureau of Economic Research.

Jentsch, Peter, Madhur Anand, and Chris T Bauch. 2020. "Prioritising COVID-19 Vaccination in Changing Social and Epidemiological Landscapes." Working Paper 2020.09.25.20201889, medRxiv.

Joint United Nations Programme on HIV and AIDS. 2020. "Fact Shet - World AIDS Day 2020." Global HIV and AIDS Statistics, Joint United Nations Programme on HIV and AIDS.

Kaiser Family Foundation. 2019. "The HIV/AIDS Epidemic in the United States: The Basics." Available at https://www.kff.org/hivaids/fact-sheet/ the-hivaids-epidemic-in-the-united-states-the-basics/.

Kermack, William Ogilvy and Anderson G McKendrick. 1927. "A Contribution to the Mathematical Theory of Epidemics." Proceedings of the Royal Society of London Series A. 115 (772):700-721.

Labgold, Katie, Sarah Hamid, Sarita Shah, Neel R. Gandhi, Allison Chamberlain, Fazle Khan, Shamimul Khan, Sasha Smith, Steve Williams, Timothy L. Lash, and Lindsay J. Collin. 2021. "Estimating the Unknown: Greater Racial and Ethnic Disparities in COVID-19 Burden After Accounting for Missing Race and Ethnicity Data." Epidemiology 32 (2):157-161. 
Li, Xue-Zhi, Junyuan Yang, and Maia Martcheva. 2020. Age Structured Epidemic Modeling, vol. 52. Springer.

Lighter, Jennifer, Michael Phillips, Sarah Hochman, Stephanie Sterling, Diane Johnson, Fritz Francois, and Anna Stachel. 2020. "Obesity in Patients Younger Than 60 Years Is a Risk Factor for COVID-19 Hospital Admission." Clinical Infectious Diseases 71 (15):896-897.

Lochner, Lance and Enrico Moretti. 2004. "The Effect of Education on Crime: Evidence from Prison Inmates, Arrests, and Self-Reports." American Economic Review 94 (1):155-189.

Machin, Stephen, Olivier Marie, and Sunčica Vujić. 2011. "The Crime Reducing Effect of Education." The Economic Journal 121 (552):463-484.

Manfredi, Piero and Alberto D'Onofrio. 2013. Modeling the Interplay Between Human Behavior and the Spread of Infectious Diseases. Springer Science and Business Media.

Marcus, Julia. 2020. "Quarantine Fatigue Is Real." The Atlantic. Available at https://www.theatlantic.com/ideas/archive/2020/05/ quarantine-fatigue-real-and-shaming-people-wont-help/611482/.

Martcheva, Maia. 2015. An Introduction to Mathematical Epidemiology, vol. 61. Springer.

McAdams, David. 2020. "Nash SIR: An Economic-Epidemiological Model of Strategic Behavior During a Viral Epidemic." Covid Economics 16:115-134.

McLaren, John and Su Wang. 2020. "Effects of Reduced Workplace Presence on Covid-19 Deaths: An Instrumental-Variables Approach." Working Paper 28275, National Bureau of Economic Research.

Murray, Eleanor J. 2020. "Epidemiology's Time of Need: COVID-19 Calls for EpidemicRelated Economics." Journal of Economic Perspectives 34 (4):105-20.

Neergaard, Lauran. 2020. "Healing is Coming": U.S. Health Workers Start Getting Vaccine." Associated Press. Available at https://apnews.com/article/ us-health-workers-coronavirus-vaccine-56df745388a9fc12ae93c6f9a0d0e81f

Papageorge, Nicholas W. 2016. "Why Medical Innovation is Valuable: Health, Human Capital, and the Labor Market." Quantitative Economics 7 (3):671-725.

Papageorge, Nicholas W., Gwyn C. Pauley, Mardge Cohen, Tracey E. Wilson, Barton H. Hamilton, and Robert A. Pollak. 2019. "Health, Human Capital and Domestic Violence.' Journal of Human Resources Forthcoming.

Papageorge, Nicholas W., Matthew V. Zahn, Michèle Belot, Eline van den Broek-Altenburg, Syngjoo Choi, Julian C. Jamison, and Egon Tripodi. 2021. "Socio-Demographic Factors Associated with Self-Protecting Behavior during the Covid-19 Pandemic." Journal of Population Economics Forthcoming. 
Poulson, Michael, Alaina Geary, Chandler Annesi, Lisa Allee, Kelly Kenzik, Sabrina Sanchez, Jennifer Tseng, and Tracey Dechert. 2021. "National Disparities in COVID-19 Outcomes Between Black and White Americans." Journal of the National Medical Association $113(2): 125-132$.

Ravi, Krithi. 2020. "Ethnic Disparities in COVID-19 Mortality: Are Comorbidities to Blame?" The Lancet 396 (10243):22.

Ray, Evan L., Nutcha Wattanachit, Jarad Niemi, Abdul Hannan Kanji, Katie House, Estee Y. Cramer, Johannes Bracher, Andrew Zheng, Teresa K. Yamana, Xinyue Xiong, Spencer Woody, Yuanjia Wang, Lily Wang, Robert L. Walraven, Vishal Tomar, Katherine Sherratt, Daniel Sheldon, Robert C. Reiner, B. Aditya Prakash, Dave Osthus, Michael Lingzhi Li, Elizabeth C. Lee, Ugur Koyluoglu, Pinar Keskinocak, Youyang Gu, Quanquan Gu, Glover E. George, Guido España, Sabrina Corsetti, Jagpreet Chhatwal, Sean Cavany, Hannah Biegel, Michal Ben-Nun, Jo Walker, Rachel Slayton, Velma Lopez, Matthew Biggerstaff, Michael A. Johansson, and Nicholas G. Reich. 2020. "Ensemble Forecasts of Coronavirus Disease 2019 (COVID-19) in the U.S." Working Paper 2020.08.19.20177493, medRxiv.

Roosa, K., Y. Lee, R. Luo, A. Kirpich, R. Rothenberg, J.M. Hyman, P. Yan, and G. Chowell. 2020. "Real-Time Forecasts of the COVID-19 Epidemic in China from February 5th to February 24th, 2020." Infectious Disease Modelling 5:256-263.

Rozenfeld, Yelena, Jennifer Beam, Haley Maier, Whitney Haggerson, Karen Boudreau, Jamie Carlson, and Rhonda Medows. 2020. "A Model of Disparities: Risk Factors Associated with COVID-19 Infection." International Journal for Equity in Health 19 (1):1-10.

Serkez, Yaryna. 2020. "The Magic Number for Reducing Infections and Keeping Businesses Open." The New York Times. Available at https://www.nytimes.com/interactive/ 2020/12/16/opinion/coronavirus-shutdown-strategies.html.

Shaman Group. 2014. "Influenza Forecast." Available at https://blogs.cuit.columbia. edu/jls106/projects/influenza-forecast/.

Simonov, Andrey, Szymon K. Sacher, Jean-Pierre H. Dubé, and Shirsho Biswas. 2020. "The Persuasive Effect of Fox News: Non-Compliance with Social Distancing During the Covid19 Pandemic." Working Paper 27237, National Bureau of Economic Research.

Smith, Aaron A, Jeremy Fridling, Danyal Ibhrahim, and Paul S Porter Jr. 2020. "Identifying Patients at Greatest Risk of Mortality Due to COVID-19: A New England perspective." Western Journal of Emergency Medicine 21 (4):785.

Smith, J Maynard and George R Price. 1973. "The Logic of Animal Conflict." Nature 246 (5427):15-18.

Taylor, Peter D and Leo B Jonker. 1978. "Evolutionary Stable Strategies and Game Dynamics." Mathematical Biosciences 40 (1-2):145-156. 
Varghese, Beena, Julie E. Maher, Thomas A. Peterman, Bernard M. Branson, and Richard W. Steketee. 2002. "Reducing the Risk of Sexual HIV Transmission: Quantifying the Per-Act Risk for HIV on the Basis of Choice of Partner, Sex Act, and Condom Use." Sexually Transmitted Diseases 29 (1):38-43.

White, Edmund. 1991. States of Desire: Travels in Gay America. Plume Books.

Wright, Austin L., Konstantin Sonin, Jesse Driscoll, and Jarnickae Wilson. 2020. "Poverty and Economic Dislocation Reduce Compliance with COVID-19 Shelter-in-Place Protocols." Journal of Economic Behavior \&3 Organization 180:544-554.

Wu, Chaomin, Xiaoyan Chen, Yanping Cai, Jia'an Xia, Xing Zhou, Sha Xu, Hanping Huang, Li Zhang, Xia Zhou, Chunling Du, Yuye Zhang, Juan Song, Sijiao Wang, Yencheng Chao, Zeyong Yang, Jie Xu, Xin Zhou, Dechang Chen, Weining Xiong, Lei Xu, Feng Zhou, Jinjun Jiang, Chunxue Bai, Junhua Zheng, and Yuanlin Song. 2020a. "Risk Factors Associated With Acute Respiratory Distress Syndrome and Death in Patients With Coronavirus Disease 2019 Pneumonia in Wuhan, China." Journal of the American Medical Association Internal Medicine 180 (7):934-943.

Wu, Renyi, Lujing Wang, Hsiao-Chen Dina Kuo, Ahmad Shannar, Rebecca Peter, Pochung Jordan Chou, Shanyi Li, Rasika Hudlikar, Xia Liu, Zhigang Liu et al. 2020b. "An Update on Current Therapeutic Drugs Treating COVID-19." Current Pharmacology Reports 6 (3):56-70.

Yang, Junyuan, Maia Martcheva, and Yuming Chen. 2016. "Imitation Dynamics of Vaccine Decision-Making Behaviours Based on the Game Theory." Journal of Biological Dynamics $10(1): 31-58$.

Zhang, Renyi, Yixin Li, Annie L. Zhang, Yuan Wang, and Mario J. Molina. 2020. "Identifying airborne transmission as the dominant route for the spread of COVID-19." Proceedings of the National Academy of Sciences 117 (26):14857-14863.

Zhu, Na, Dingyu Zhang, Wenling Wang, Xingwang Li, Bo Yang, Jingdong Song, Xiang Zhao, Baoying Huang, Weifeng Shi, Roujian Lu, Peihua Niu, Faxian Zhan, Xuejun Ma, Dayan Wang, Wenbo Xu, Guizhen Wu, George F. Gao, and Wenjie Tan. 2020. "A Novel Coronavirus from Patients with Pneumonia in China, 2019." New England Journal of Medicine 382 (8):727-733. 


\section{$5 \quad$ Tables and Figures}

Table 1: Summary Statistics: MACS Dataset

\begin{tabular}{|c|c|c|c|c|c|}
\hline & (1) & $(2)$ & $(3)$ & (4) & $(5)$ \\
\hline & Total & \multicolumn{2}{|c|}{ HIV-negative } & \multicolumn{2}{|c|}{ HIV-positive } \\
\hline & & $\begin{array}{c}\text { pre- } \\
\text { HAART }\end{array}$ & $\begin{array}{c}\text { post- } \\
\text { HAART }\end{array}$ & $\begin{array}{c}\text { pre- } \\
\text { HAART }\end{array}$ & $\begin{array}{c}\text { post- } \\
\text { HAART }\end{array}$ \\
\hline HIV Positive & 0.41 & 0.00 & 0.00 & 1.00 & 1.00 \\
\hline Age & 44 & 42 & 49 & 40 & 46 \\
\hline College+ & 0.63 & 0.67 & 0.68 & 0.55 & 0.58 \\
\hline CD4 Count & 725 & 1066 & 971 & 406 & 483 \\
\hline Death & 0.02 & 0.002 & 0.003 & 0.05 & 0.01 \\
\hline Seroconversion & 0.003 & 0.00 & 0.00 & 0.01 & 0.004 \\
\hline Moderate \& Risky & 0.56 & 0.54 & 0.60 & 0.55 & 0.56 \\
\hline Risky & 0.04 & 0.03 & 0.05 & 0.04 & 0.06 \\
\hline Moderate & 0.51 & 0.50 & 0.55 & 0.51 & 0.50 \\
\hline Any Medication & 0.28 & 0.00 & 0.00 & 0.55 & 0.79 \\
\hline HAART & 0.11 & 0.00 & 0.00 & 0.00 & 0.59 \\
\hline Ailments & 0.28 & 0.17 & 0.20 & 0.41 & 0.42 \\
\hline Full time work & 0.74 & 0.81 & 0.76 & 0.70 & 0.61 \\
\hline Income (6 months) & 18,465 & 18,347 & 21,031 & 16,605 & 18,464 \\
\hline
\end{tabular}

Notes: Summary statistics are based on 47,763 bi-annual person-visit observations from 3,999 men between 1990 and 2003. The post-HAART period starts in mid-1995. Death is defined as the probability that an individual leaves the sample due to death between the current period and the next. Seroconversion is defined as the probability that an HIV - individual in the current period is HIV + in the following visit. High risk sex means individuals engage in anal sex with multiple partners without consistent condom usage. Moderate risk sex means limiting anal sex to one partner or using condoms consistently. Ailments include sweats, fever, diarrhea, fatigue, and headache. 
Table 2: Model Objects

\begin{tabular}{|c|c|c|}
\hline Parameter & Source & Notation \\
\hline $\begin{array}{l}\text { Disutility of Work } \\
\text { Health } \\
\text { Age } \\
\text { Pre-existing Condition } \\
\text { Socioeconomic Characteristics } \\
\text { Cost of Protection }\end{array}$ & $\begin{array}{l}\text { Estimate } \\
\text { Estimate } \\
\text { Estimate } \\
\text { Estimate } \\
\text { Estimate } \\
\text { Estimate }\end{array}$ & $\begin{array}{l}\theta_{W} \\
\theta_{h} \\
\theta_{A, j} \\
\theta_{B, j} \\
\theta_{S, j} \\
\theta_{\hat{d}} \\
\end{array}$ \\
\hline $\begin{array}{l}\text { Healthy to Infected: Not Taking Action } \\
\text { Healthy to Infected: Taking Action } \\
\text { Infected, Asymptomatic, and Unaware to Aware } \\
\text { Infected, Asymptomatic, to Symptomatic } \\
\text { Infected to Healthy Without Immunity } \\
\text { Infected to Healthy With Immunity } \\
\text { Death }\end{array}$ & $\begin{array}{l}\text { Data, Reduced Form, or Estimate } \\
\text { Data, Calibrate } \\
\text { Data, Calibrate } \\
\text { Data, Calibrate } \\
\text { Data } \\
\text { Data } \\
\text { Data, Calibrate }\end{array}$ & $\begin{array}{l}P_{t}\left(h_{t+1}=1 \mid h_{t}=0, X_{t}, d_{t}, \bar{d}_{t}, \bar{\lambda}_{t}\right) \\
\omega \\
\tau\left(X_{i t}\right) \\
\zeta\left(X_{i t}\right) \\
\psi \\
\rho \\
P\left(\delta \mid X_{i t}\right)\end{array}$ \\
\hline $\begin{array}{l}\text { AR(1) Coefficient } \\
\text { Susceptible: Taking Action } \\
\text { Susceptible: Not Taking Action } \\
\text { Death Rate } \\
\text { Geographic Factors }\end{array}$ & $\begin{array}{l}\text { Reduced Form } \\
\text { Reduced Form } \\
\text { Reduced Form } \\
\text { Reduced Form } \\
\text { Reduced Form } \\
\end{array}$ & $\begin{array}{l}\phi \\
\alpha_{1} \\
\alpha_{2} \\
\delta \\
\gamma\end{array}$ \\
\hline $\begin{array}{l}\text { Labor Income } \\
\text { Labor Income Shock Variance } \\
\text { Non-Labor Income } \\
\text { Non-Labor Income Shock Variance } \\
\text { Consumption }\end{array}$ & $\begin{array}{l}\text { Reduced Form/Match } \\
\text { Calibrate } \\
\text { Reduced Form/Match } \\
\text { Calibrate } \\
\text { Reduced Form/Match }\end{array}$ & $\begin{array}{l}f\left(X_{i t}\right) \\
\sigma_{v^{w}}^{y} \\
g\left(X_{i t}\right) \\
\sigma_{v^{b}}^{b} \\
h\left(y_{i t}, b_{i t}\right)\end{array}$ \\
\hline
\end{tabular}

Notes: This table summarizes objects in the structural model that I present that are either estimated or calibrated. The first column describes the parameter. The second column describes whether the parameter is estimated, recovered from data, or calibrated to match an external source. The third column prints the notation used for that parameter from the model write up. 


\section{Model Estimation and Data Requirements}

I will briefly describe how the structural parameters of this model can be estimated via maximum likelihood. Some objects within the model that do not vary with the structural parameter estimates are computed or calibrated in a first stage. These objects are the health transition processes (i.e., symptom and immunity development, recovery, and death) and the parameters of the aggregate infection rate.

In the second step, these processes are used to recover the structural parameters iteratively. The algorithm begins with an initial guess for the conditional choice probabilities for each choice $j$ by health status an individual may make and the aggregate infection rate. Given a candidate set of parameters $\Xi$, compute the current period payoffs for each choice $j$ and combinations of the state variables. Next, given the choice probabilities for action $j$ by health status and the aggregate infection rate for the current period, generate an estimate for $\bar{\lambda}_{t+1}$. From here, use the estimated $\bar{\lambda}_{t+1}$ and the transition processes to compute the net-of-errors value functions $\left(\bar{V}_{j t}\right)$ and use them to recover the choice probabilities implied by the model $\left(\phi_{j t}\right)$.

The choice probabilities $\phi_{j t}$ reflect optimal behavior given the structural parameters and the initial guesses for the choice probabilities conditional on health status. When $\phi_{j t}$ is aggregated to the market-level, these probabilities may differ from the initial guesses used for the expectations in calculating the net-of-errors value functions. As a result, I have calculated optimal behavior with beliefs that are not consistent with the equilibrium behavior implied by $\phi_{j t}$. In this case, the choice probabilities $\phi_{j t}$ are aggregated and used to update the initial conditional choice probabilities. The second step is repeated until the equilibrium constraint is satisfied. In other words, the second step is repeated until optimal behavior implied by the model $\phi_{j t}$ is consistent with the agent's expectations about optimal behavior (i.e., the choice probabilities conditional on health status). This process is the calculation for a given set of structural parameters $\Xi$. The process repeats for different candidate $\Xi$ until the likelihood 
function is maximized.

To estimate this model, individual level survey data during an infectious disease outbreak is required. These data ought to include information about the individual's health status, other characteristics, and their behavioral choices, ideally both work and pandemic related. Beliefs and perceptions about the pandemic or infection rate would also be valuable. These data should include geographic details for the respondents so that they can be supplemented with other data sources to provide information about the environment where the respondent lives and works. From these data, one can recover empirical estimates of the conditional choice probabilities as well as the transition processes that will be included in the model. Epidemiological data about the infectious disease in question may also be necessary to estimate this model. For example, the SIR model presented in my framework requires information about the rates of recovery and immunity development, among others. To the extent these transitions are not readily recoverable from survey data, it may be necessary to get this information from medical sources to calibrate them. 